\title{
Kernos
}

Revue internationale et pluridisciplinaire de religion grecque antique

$28 \mid 2015$

Varia

\section{Zeus Polieus à Athènes}

Les Bouphonies et au-delà

\section{Sylvain Lebreton}

\section{(2) OpenEdition}

1 Journals

\section{Édition électronique}

URL : http://journals.openedition.org/kernos/2330

DOI : $10.4000 /$ kernos. 2330

ISSN : 2034-7871

Éditeur

Centre international d'étude de la religion grecque antique

\section{Édition imprimée}

Date de publication : 1 octobre 2015

Pagination : 85-110

ISBN : 978-2-87562-055-2

ISSN : 0776-3824

\section{Référence électronique}

Sylvain Lebreton, «Zeus Polieus à Athènes », Kernos [En ligne], 28 | 2015, mis en ligne le 01 octobre 2017, consulté le 01 mai 2019. URL : http://journals.openedition.org/kernos/2330 ; DOI : 10.4000/ kernos. 2330

Ce document a été généré automatiquement le 1 mai 2019.

Kernos 


\title{
Zeus Polieus à Athènes
}

\author{
Les Bouphonies et au-delà
}

\author{
Sylvain Lebreton
}

\begin{abstract}
Cet article trouve sa source dans ma thèse de doctorat (Surnommer Zeus. Contribution à l'étude des structures et des dynamiques du polythéisme attique à travers ses épiclèses, de l'époque archaïque au Haut-Empire, Université Rennes 2, 2013), actuellement en cours de publication (à paraitre dans un prochain supplément Kernos), dans le cadre d'un mandat post-doctoral BeIPD-COFUND à l'Université de Liège. Sauf indication contraire, les dates sont entendues avant notre ère, l'Acropole et l'Agora (avec une majuscule) sont celles d'Athènes. Pour les sources littéraires, sauf mention contraire, on se réfere aux éditions de la CUF, à l'exception de: Scholies anciennes à Aristophane, Nuées (éd. HOLWERDA); Tzetzès à Aristophane, Nuées (éd. HOLWERDA); Scholies à Démosthène (éd. DILTS); Hésychios (éd. LATTE); Photios, Lexique (éd. THEODORIDIS); Souda (éd. ADLER). Pour le détail des attestations des épiclèses évoquées ici sans référence, je renvoie à la $B D E G$. Je remercie vivement Pierre Brulé, Vinciane Pirenne-Delforge et le lecteur anonyme de Kernos pour leurs relectures attentives et leurs précieuses remarques.
\end{abstract}

1 Giove Polieo in Athene: c'est ainsi qu'Otto Jahn titrait il y a tout juste 150 ans un mémoire essentiellement consacré, déjà, aux Dipolies et aux Bouphonies. S'ouvrait ainsi un long et riche débat historiographique sur l'interprétation de cette fête et de la mise à mort du bœuf qui en constituait probablement le rituel central. Or, si ce débat a abondamment nourri la réflexion sur les questions sacrificielles ${ }^{1}$, il a paradoxalement occulté la figure du Zeus Polieus athénien auquel ces cérémonies étaient consacrées. Après un siècle et demi de production scientifique, force est de constater, en effet, que toute la lumière n'a pas été faite sur le rôle et la place de ce Zeus au sein du panthéon attique. Alors que l'interprétation des Bouphonies, et notamment de sa relation par Porphyre dans son traité De l'abstinence (De Abstinentia), est toujours fort discutée, on s'accorde en effet à voir dans ce Zeus « de la polis» le protecteur de la cité, la signification de son épiclèse ne semblant pas poser problème ${ }^{2}$.

2 Or, une telle signification ne va pas de soi, ne serait-ce qu'en raison de la polysémie du terme polis lui-même, qui peut en effet désigner la communauté humaine (la polis-cité) tout autant que l'acropole (la polis-citadelle) ${ }^{3}$. Un premier niveau d'interrogation consiste donc à se demander si Zeus était Polieus en Attique parce qu'il était conçu comme un 
protecteur de la cité, un garant de son unité ou bien parce que son culte prenait place sur la ville haute ${ }^{4}$, autrement dit, s'il faut donner à l'épiclèse un sens fonctionnel ou topographique. En l'espèce, les deux acceptions peuvent se combiner: on pense évidemment à l'Athéna Polias athénienne, archétype de la divinité " poliade », qui occupe une fonction tutélaire depuis son séjour acropolitain 5 . Ce serait toutefois aller un peu vite que de transposer ce double caractère au Polieus, d'autant plus que l'association de ce dernier avec la Polias semble, à Athènes du moins, difficile à saisir. Si le Polieus est indéniablement acropolitain tout en tenant bien un rôle " politique » (entendu au sens de « relatif à la polis »), il n'est pas une Polias au masculin.

3 En plus de cette polysémie intrinsèque, il faut également tenir compte du fait que le sens à donner à cette épiclèse - comme à toutes les autres - ne coïncide pas nécessairement avec sa signification littérale: il peut la recouvrir parfaitement, mais aussi potentiellement la dépasser, la restreindre, s'en écarter même. Il est ainsi nécessaire, pour prendre la pleine mesure des représentations que les anciens Grecs - les Athéniens en l'occurrence ${ }^{6}$ - se faisaient d'une figure divine désignée par une épiclèse donnée, de prendre en compte, quand c'est possible, l'ensemble des occurrences documentaires dans laquelle celle-ci se retrouve, et de questionner à chaque fois l'identité et les motivations des acteurs qui s'adressent à elle. À l'aune d'un tel examen, il apparaît que le champ sémantique recouvert par l'épithète Polieus est particulièrement riche en Attique: il inclut évidemment la situation acropolitaine, qui n'est pas sans lien avec le caractère élevé de Zeus ; il touche aux questions agricoles, même si l'intervention du Polieus dans ce champ reste difficile à définir avec précision; il se réfère aussi à la polis-cité, mais la nature de la protection attendue de Zeus ainsi désigné doit être déterminée. En effet, dans un cadre polythéiste aussi riche que celui des Athéniens et dans un domaine aussi central que celui de la cité, le Polieus n'intervient pas seul. Par conséquent, sa mise en relation avec les autres puissances divines intervenant parallèlement, conjointement, concurremment dans ce champ politique devrait permettre de faire ressortir l'étendue de ses fonctions et l'éventuelle singularité de son mode d'intervention.

4 Il s'agira dès lors de reprendre l'ensemble du dossier relatif au culte de Zeus Polieus en Attique, à l'échelle de la cité et des dèmes ${ }^{7}$, sur l'ensemble de la période pour laquelle son culte est attesté, à savoir du tournant des $\mathrm{VI}^{\mathrm{e}}-\mathrm{V}^{\mathrm{e}}$ siècles jusqu'au $\mathrm{III}^{\mathrm{e}}$ siècle de notre ère. La question des Bouphonies ne sera pas négligée, mais d'autres sources que le seul Porphyre seront mises à contribution, en particulier une glose d'Hésychios méconnue qui apporte pourtant un contrepoint fort intéressant au De Abstinentia. Du reste, on ne pourra prendre la pleine mesure du rôle du Polieus athénien qu'en mettant ces données en perspective, en les confrontant aux autres figures du dieu connues en Attique, notamment en ce qui concerne son positionnement singulier par rapport à Athéna (Polias). Si ce Zeus est bien un dieu De la cité, il convient de se demander dans quelle mesure il a pu être conçu comme son protecteur direct, et si d'autres Zeus athéniens n'ont pas tenu ce rôle de façon plus manifeste et plus active.

\section{Zeus Polieus sur l'Acropole : cadres et portée du culte politique}

$5 \quad$ Les principales informations dont on dispose au sujet de Zeus Polieus concernent son culte à l'échelle politique, qui avait pour principaux cadres son sanctuaire acropolitain et la fête des Dipolies. Ces données sont toutefois éparses et ne permettent d'en reconstituer 
l'organisation que de façon partielle. Leur examen permettra néanmoins de proposer quelques hypothèses sur les finalités de ce culte.

\section{L'espace : le sanctuaire acropolitain}

6 S'il est assuré que Zeus Polieus avait son principal lieu de culte sur l'Acropole, les données relatives à ce sujet sont plutôt maigres. Pausanias fait seulement état d'une statue et d'un autel du dieu à cet endroit ${ }^{8}$. On a toutefois proposé de localiser le sanctuaire au nord-est du Parthénon, au point culminant de l'Acropole $(156 \mathrm{~m})$, sur une plate-forme taillée dans la roche qui semble avoir connu deux phases d'aménagement : une première à la fin de l'époque archaïque et une autre lors du troisième quart du v viècle, probablement dans le cadre des grands travaux péricléens. Les structures comportent deux parties : un enclos trapézoïdal à ciel ouvert (de $26 \times 16,5$ à $42 \times 35 \mathrm{~m}$ selon les estimations) et une enceinte polygonale $(18 \times 25 \mathrm{~m})$ enserrant un petit temple orienté au nord $(4,5 \times 7 \mathrm{~m})$ comportant une fosse dans la cella et devant lequel se trouve une longue table à offrandes. Compte tenu de l'état des vestiges, ces données sont toutefois difficiles à interpréter : l'hypothèse de la présence d'étables en bois pouvant accueillir du bétail - celui des Dipolies notamment - un temps formulée, reste fragile; et s'il est possible que l'enclos trapézoïdal ait pu servir à parquer des bêtes, celles-ci n'avaient peut-être aucun lien avec le culte de Zeus Polieus: il n'est pas impossible qu'il s'agisse des bœufs amenés sur l'Acropole pour les Panathénées. Du reste, il n'est même pas certain que les deux parties de l'ensemble - l'enclos et le temple - fussent liées dans leur utilisation cultuelle ${ }^{9}$. En revanche, il y a tout lieu de penser que l'enceinte qui se dressait autour du temple devait permettre de contrôler les mouvements des bêtes qui circumambulaient lors des Dipolies, en plus de marquer matériellement et symboliquement les limites du lieu.

7 Quoi qu'il en soit, c'est sur son autel acropolitain que le dieu recevait des sacrifices, notamment ceux offerts par la cité lors des Dipolies ou encore par les démotes d'Erchia, en Métageitniôn. On n'a conservé que peu de traces des offrandes d'autres types qui lui étaient consacrées : les inventaires de l'Hécatompédon répertorient entre 428/7 et 394/3 un karchèsion d'argent d'un poids de 200 drachmes lui appartenant ${ }^{10}$, ce qui laisse entendre que ses richesses, dont on peine à mesurer l'importance ${ }^{11}$, n'étaient pas entreposées dans son sanctuaire. On ne connaît ni l'occasion, ni les motivations de l'offrande de ce vase, pas plus que de celle de la seule dédicace adressée à Zeus Polieus retrouvée en Attique. L'état lacunaire de cette dernière ne permet pas d'en saisir toute la portée : de l'identité du dédicant, notamment, on ne peut déterminer que le démotique ( Paianieus), ce qui, isolé de tout contexte, et en l'absence d'autres exemples, ne permet guère d'attester que son statut de citoyen ${ }^{12}$.

8 La seule autre source d'information sur les espaces liés au culte du dieu à Athènes ne provient pas de l'Acropole, mais du secteur du cimetière du Cynosarges (Diamantopoulou 10), où a été récemment mise au jour une borne portant l'inscription suivante, datable de la seconde moitié $\mathrm{du} \mathrm{v}^{\mathrm{e}} \mathrm{s} .^{13}$ :

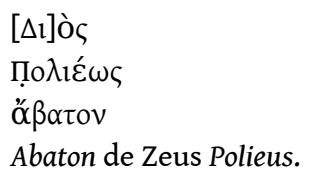

9 Compte tenu de sa nature, une telle borne n'était sans doute pas intégrée au cimetière du Cynosarges lui-même. Il est possible qu'il s'agisse d'une pierre errante ; le cas échéant, 
elle pourrait provenir du sanctuaire acropolitain du Polieus. Toutefois, peu d'inscriptions de ce type ont été retrouvées sur l'Acropole même (versants mis à part), où il était peu utile de délimiter les espaces sacrés. À la suite de l'éditeur du texte, il faudrait donc rejeter cette hypothèse et tenir le lieu de découverte de ce monument - dans le secteur du Cynosarges, probablement dans le dème de Diomeia - comme signifiant. Selon lui, la forme du support implique en effet que cette borne était située au bord d'une rue animée, d'un axe de circulation; le texte tend à le confirmer qui défend de fouler l'espace sacré ainsi délimité en le définissant comme abaton. Cette interdiction ne s'expliquerait donc pas uniquement par des raisons théologiques, mais aussi par les contraintes de l'environnement du sanctuaire ${ }^{14}$ : l'objectif serait en l'occurrence de préserver son intégrité des empiètements, qu'ils soient occasionnels (dus à la circulation) ou permanents (procédant de conflits d'usage d'un espace dense et convoité). Plus précisément, la proximité du cimetière - et les risques de souillure qui en résultent pourrait expliquer le caractère explicite de l'inscription : comme tout espace consacré à une divinité, celui-ci doit être protégé de l'impureté ${ }^{15}$. Il est probable que cet abaton était lié au sanctuaire acropolitain du Polieus ${ }^{16}$, sans que la nature du lien entre ces deux espaces puisse être établie avec certitude : le premier était-il une simple dépendance du second ou bien accueillait-il le cadre d'une activité cultuelle propre? À ce stade, on ne peut trancher la question sans avoir examiné le déroulement des rites accomplis en l'honneur du dieu.

\section{Dipolies et Bouphonies : organisation, déroulement et acteurs}

Les Dipolies, et le rite des Bouphonies qui en faisait partie, constituaient le temps fort du culte athénien de Zeus Polieus. La fête a déjà été abondamment commentée: on se contentera donc d'en retracer brièvement le déroulement, d'apporter quelques éclaircissements sur la sélection des bêtes ainsi que sur ses acteurs, pour mieux revenir sur ses enjeux. Sur tous ces aspects, il faudra rester conscient des fragilités et des disparités chronologiques d'un ensemble documentaire composé pour l'essentiel d'inscriptions de l'époque classique, pour la plupart très fragmentaires, et des récits d'Androtion (transmis par des scholies et gloses lexicographiques), de Pausanias, d'Élien et de Porphyre (inspiré de Théophraste) ${ }^{17}$.

11 En ce qui concerne le rituel, on est ainsi réduit à supposer une relative stabilité sur le temps long. Les Dipolies étaient célébrées avec faste chaque année le 14 (ou le 16) Skirophoriôn ${ }^{18}$, tout à la fois au cœur de l'été et à la fin de l'année. Elles comportaient une procession, dont le point de départ est inconnu (l'abaton du Cynosarges, qui aurait alors eu la fonction d'une « étable sacrée " pour les bêtes ?), mais qui aboutissait au sanctuaire de l'Acropole ${ }^{19}$. Là, un (des ?) pelanos (-oi) et / ou des grains étaient déposés sur l'autel ou la table de bronze du dieu, autour duquel ou de laquelle on faisait déambuler des bœufs ${ }^{20}$, jusqu'à ce que l'un d'entre eux se penche vers les victuailles. L'animal était alors abattu, égorgé, dépecé et sa viande était consommée collectivement; la peau était bourrée de foin pour former une effigie de la bête à laquelle on attelait une charrue et on faisait mimer le labour. Enfin avait lieu le procès des instruments sacrificiels : selon Pausanias, c'est la hache (pelekus) qui avait servi à abattre le bœuf qui était traduite en justice au Prytanée et relaxée ; selon Porphyre, c'est le couteau (machaira) utilisé pour l'égorgement qui était reconnu pour seul coupable et jeté à la mer. 
12 Cette possible reconstitution reste grevée de plusieurs incertitudes. Les premières concernent le nombre, la fourniture et la sélection des bêtes. Pausanias n'évoque que le bœuf qui a touché aux offrandes de l'autel, en précisant qu'il avait été préparé et gardé pour le sacrifice; Porphyre précise que si un seul animal est mis à mort, plusieurs déambulaient autour de l'autel et étaient sélectionnés à cet effet ${ }^{21}$. Il semblerait donc qu'une présélection avait lieu en amont, avant la procession et la circumambulation des bœufs, et que le choix final était déterminé par la consommation des victuailles déposées sur l'autel. L'entrée du calendrier dit « de Nikomachos» (fin du v siècle) consacrée aux Dipolies indique que la cité fournissait les six premiers, ce qui laisse entendre que d'autres étaient fournis par d'autres acteurs, probablement les dèmes ${ }^{22}$. Le règlement cultuel des Scambonides (ca.460) prescrit en effet de fournir pour les Dipolies et les Panathénées le nécessaire pour le sacrifice et d'effectuer le partage sur l'agora du dème ; le texte est toutefois trop lacunaire pour savoir si ce "nécessaire pour le sacrifice" incluait des bêtes ${ }^{23}$. Si les restitutions et interprétations proposées pour ces deux inscriptions s'avéraient exactes, cela impliquerait que la cité et les dèmes contribuaient de conserve à la fourniture des bêtes pour les Dipolies, du moins au ve siècle.

Sur ce point, le parallèle - établi depuis longtemps déjà - entre la fête athénienne et le sacrifice de Batromios au Zeus Polieus de Cos peut s'avérer éclairant ${ }^{24}$. Lors de ce dernier en effet, différentes associations infra-politiques (notamment les tribus, elles-mêmes divisées en chiliastyes) fournissaient des bœufs qui étaient ensuite mêlés et par conséquent choisis indépendamment de leur provenance. Un tel processus peut légitimement être lu comme le moyen rituel de souder les différentes subdivisions de la communauté politique, tout particulièrement dans le contexte de la seconde moitié du Iv ${ }^{\mathrm{e}}$ siècle, immédiatement postérieur au synœecisme de l'île (366) : par ce sacrifice, le Polieus de Cos aurait pour fonction de garantir l'unité de la cité. Bien entendu, on se gardera de transposer directement cette interprétation aux Dipolies athéniennes ${ }^{25}$. Néanmoins, le hasard laissé dans le choix de l'animal mis à mort lors des Bouphonies et le fait que les viandes étaient probablement partagées entre les démotes laissent supposer que les Dipolies pouvaient avoir ce même caractère unificateur. En outre, la mention simultanée de cette fête et de celle des Panathénées dans le règlement des Scambonides invite à donner à la première la même signification unificatrice qu'à la seconde ${ }^{26}$. On le verra, les autres composantes du culte du Polieus en Attique tendent à confirmer que c'était là une fonction récurrente du dieu.

Le tableau que l'on peut dresser des acteurs des Dipolies conserve également de nombreuses zones d'ombre. Certes, les variations entre les différentes versions du récit étiologique qui concernent l'identité du premier sacrificateur des Bouphonies, dénommé Sôpatros ou Diomos chez Porphyre, Thaulôn chez Androtion ${ }^{27}$ et même Brabrôn (sic) chez Tzetzès (qui affirme pourtant s'appuyer sur le même Androtion) ${ }^{28}$ sont banales. En l'occurrence, elles reflètent le souci, en un temps et un lieu donné, d'associer au rite une localité (Braurôn ?), un dème (Diomeia ${ }^{29}$ ) ou de souligner le rôle qu'y tient un genos (les Thaulônidai; les *Boutypoi, qui auraient Sôpatros pour ancêtre, selon Porphyre). En revanche, l'articulation entre ces personnages mythiques, les lieux ou les associations auxquels ils sont liés et les acteurs «historiques » du rituel des Dipolies et Bouphonies présente davantage de difficultés ${ }^{30}$. Il semblerait que les hérauts évoqués dans le calendrier de Nikomachos, probablement issus du genos des Kentriadai, faisaient déambuler les bêtes autour de l'autel ${ }^{31}$, les hiéropes étant pour leur part en charge de la procession qui précédait ${ }^{32}$. Le boutupos (ou bouphonos, ou boutès), préposé à la mise à mort 
du bœuf, était quant à lui vraisemblablement recruté au sein du genos des Thaulônidai ${ }^{33}$, peut-être dès son apparition dans la documentation au tournant des $\mathrm{VI}^{\mathrm{e}}-\mathrm{v}^{\mathrm{e}}$ siècles ${ }^{34}$, ou au plus tard à l'époque d'Androtion (actif au IV siècle) qui le premier mentionne Thaulôn, l'ancêtre mythique du genos. En dépit de la remarque de Pausanias, qui fait du bouphonos un des hiereis du dieu, il faut sans doute distinguer cette fonction de celle du prêtre de Zeus Polieus ${ }^{35}$. En effet, les sources épigraphiques n'associent jamais ce dernier aux Dipolies ou à quelle qu'association (genos ou autre) que ce soit et n'explicitent pas davantage ses prérogatives ${ }^{36}$; ce sacerdoce paraît pourtant figurer parmi les plus prestigieux de la cité, du moins aux époques hellénistique et impériale, puisque son détenteur jouissait alors d'un des meilleurs sièges de proédrie au théâtre de Dionysos ${ }^{37}$. S'il fallait lui attribuer un rôle rituel lors des Dipolies, ce pourrait être l'égorgement de la bête au couteau, distinct, chez Porphyre, de l'abattage à la hache ${ }^{38}$; il va sans dire qu'une telle hypothèse reste très fragile.

En revanche, il est certain qu'une prêtresse participait aux Dipolies, puisque le calendrier de Nikomachos prévoyait des émoluments (apometra) à cet effet ${ }^{39}$. L'ellipse sur l'identité de la divinité qu'elle desservait invite à supposer qu'il puisse s'agir de celle d'Athéna ( Polias), ce que tendrait à confirmer une scholie qui fait des Bouphonies une fête consacrée à cette déess $\mathrm{e}^{40}$. Néanmoins, on ne sait rien de plus du rôle tenu par la prêtresse à cette occasion.

Il est possible que la répartition des rôles et fonctions rituelles lors des Dipolies ait évolué de la fin du $\mathrm{VI}^{\mathrm{e}}$ siècle à l'époque impériale, et notamment que le nombre et la désignation des officiants ait varié en fonction de l'investissement de tel ou tel type ou groupe d'acteurs (les genè en premier lieu), à une époque donnée. La division des tâches dans le récit de Porphyre en rend probablement compte: pour ménager une place à chacun (à chaque genos ou à plusieurs officiels au sein d'un même genos), on décompose le rituel de façon plus précise (en distinguant celui qui présente la hache de celui qui frappe la bête e.g.) et les héros fondateurs sont associés au mythe en conséquence. Toutefois, la sédimentation documentaire des sources littéraires, associée au caractère lacunaire des inscriptions disponibles, ne permet pas de décrire cette possible évolution avec précision. En particulier, on ne peut savoir si les mutations qu'ont connues les genè au cours de l'époque hellénistique ont eu une incidence sur l'organisation des Dipolies ${ }^{41}$. Si cette hypothèse devait être retenue, elle pourrait témoigner du maintien de la fonction unificatrice de la fête en dépit des évolutions politico-sociales qu'a connues Athènes : en effet, alors que les inscriptions des $\mathrm{V}^{\mathrm{e}}$ et IV $\mathrm{V}^{\mathrm{e}}$ siècles rendent compte de la complémentarité des contributions de la cité et des dèmes, le De Abstinentia mettrait en évidence la distribution des rôles rituels entre différents genè. Autrement dit, si la cité n'est plus la même à l'époque de Porphyre qu'à l'époque classique, son unité semble toujours garantie par la force centripète du Polieus.

Bien entendu, c'est dans une optique morale, et non sociale, que Porphyre donne le détail des différents acteurs du mythe et du rite des Bouphonies, ce qui lui permet de développer la question du rejet de la culpabilité de la mise à mort du bœuf. C'est sur cette portée des Dipolies qu'il convient désormais de se pencher.

\section{Retour sur l'interprétation des Dipolies et Bouphonies}

18 À en croire le récit de Porphyre (et ceux, plus succincts, des autres auteurs), le cœur du rite et du mythe qui le justifie repose sur le problème qui naît du sacrifice du bœuf qui 
laboure : il s'agirait d'évacuer la culpabilité que suscite cette mise à mort en la reportant soit sur l'animal lui-même (coupable d'avoir mangé les offrandes déposées sur l'autel), soit sur les instruments du sacrifice. Un premier niveau de lecture peut y voir la résolution d'un problème d'économie sacrée : le rituel permet de valider le sacrifice d'un animal potentiellement utile économiquement, d'encadrer la tension liée au risque de se priver de sa force de travail. Walter Burkert, pour sa part, fait des Bouphonies une cérémonie de dissolution : la date des Dipolies, en Skirophoriôn, c'est-à-dire à la fin de l'année agricole, ainsi que leur teneur grotesque (le simulacre de labour avec le faux bœuf rempli de paille, le procès des instruments de sacrifices...) le suggèrent ${ }^{42}$; cette dimension n'était sans doute pas absente de la fête.

Une autre option a été de faire de la fête une illustration de la répugnance des Grecs à mettre à mort des êtres animés : les accusations successives des acteurs du culte et la condamnation finale du couteau et / ou de la hache lors des Bouphonies participeraient ainsi de la " comédie de l'innocence ", théorisée par Karl Meuli, qui serait caractéristique du sacrifice grec et qui permettrait de se dégager de la culpabilité du «meurtre » des animaux ${ }^{43}$. Si cette interprétation a connu un grand retentissement, elle n'a pas manqué de soulever un certain nombre de critiques justifiées: on ne doit pas perdre de vue, notamment, que la relation détaillée du mythe de Diomos / Sôpatros et du déroulement des Bouphonies dans le De Abstinentia sert d'illustration à un plaidoyer contre la consommation de la viande, et donc du sacrifice sanglant dans son ensemble ; cette vision végétarienne militante n'était évidemment pas celle des Athéniens qui consommaient la chair des bêtes après les avoir sacrifiées ${ }^{44}$. Le tout est de savoir si, pour ces derniers, les Bouphonies ne posaient que le problème du sacrifice du bœuf qui laboure ou si elles cristallisaient la tension prétendument liée à tout sacrifice sanglant ${ }^{45}$.

Par ailleurs, on ne peut faire abstraction de l'identité du destinataire divin des Dipolies. En Attique, plusieurs Zeus montagnards ou élevés ne recevaient pas de sacrifices sanglants avec consommation de viande : des holocaustes semblent attestés pour les Zeus de l'Hymette et du Parnès à l'époque archaïque, ainsi que pour l'Epôpetès d'Erchia au $\mathrm{IV}^{\mathrm{e}}$ siècle ${ }^{46}$; le Polieus lui-même recevait ce type de sacrifice, lors des Pr(o)èrosia de Thorikos (cf. infra) et peut-être même lors des Dipolies, si l'on en croit le calendrier de Nikomachos. Par ailleurs, l'Hupatos ne recevait sur son autel de l'Acropole aucun être vivant en offrande, mais seulement des gâteaux (pemmata ou pelanoi) ${ }^{47}$. Il serait ainsi possible d'interpréter le Polieus comme un pendant de ce dernier, leur deux cultes reflétant ainsi la pluralité des procédures sacrificielles athéniennes, porteuse de complémentarité mais aussi de possibles contradictions ${ }^{48}$. La situation du Polieus et de l'Hupatos sur l'Acropole, tout comme leurs épiclèses qui soulignent leur position éminente, pourrait ainsi faire sens : au cœur de la cité, en son point culminant théologique, ces deux Zeus subliment peut-être les sacrifices consacrés aux autres Zeus des sommets de l'Attique; les Bouphonies justifieraient alors la transposition des holocaustes montagnards archaïques en sacrifices à banquet, ce à quoi correspondrait l'implantation de leur destinataire sur l'Acropole, comme Polieus ${ }^{49}$. Cette hypothèse, si elle devait être validée, mettrait particulièrement en relief l'acception topographique de l'épiclèse.

21 Du reste, l'attention - légitime - dont le récit de Porphyre a fait l'objet a peut-être eu pour effet négatif d'occulter une autre interprétation possible des Dipolies et Bouphonies, plus politique ou du moins plus éclairante sur le positionnement du Polieus au sein du polythéisme attique. De fait, il existait une autre étiologie de la fête, sensiblement différente de celle que rapportent le De Abstinentia et les autres sources évoquées 
jusqu'ici. Transmise par une glose d'Hésychios, elle n'a guère été mise à contribution dans les études antérieures sur le sujet :

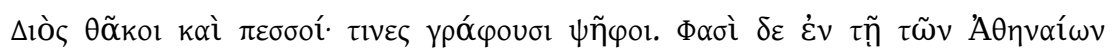

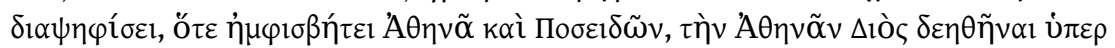

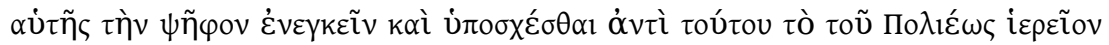

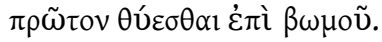

Thakoi (sièges) et pessoi (jetons) de Zeus : certains écrivent psèphoi (jetons de vote). On dit que lors du scrutin des Athéniens, alors qu'Athéna et Poséidon se disputaient, Athéna demanda à Zeus de porter son suffrage en sa faveur, en échange de quoi elle lui promit que le premier hiereion serait sacrifié sur l'autel du Polieus ${ }^{50}$.

Cette notice fait référence à la dispute entre Athéna et Poséidon pour l'obtention de la première place dans le panthéon athénien. Ce mythe bien connu est rapporté par plusieurs auteurs qui s'accordent tous sur la victoire de la déesse, mais qui divergent sur l'identité des arbitres du différend: il s'agirait ainsi de l'ensemble des Athéniens selon Varron, des douze dieux selon le Pseudo-Apollodore ${ }^{51}$. Si la version transmise par Hésychios se rapproche de la seconde tradition, elle est originale en ce qu'elle explicite la participation de Zeus au scrutin. On est peu surpris de constater que le père vote pour sa fille plutôt que pour son frère; on pourrait l'être davantage d'apprendre qu'il ne le fait pas gratuitement : c'est en effet en promettant à Zeus qu'il recevra le premier hiereion sur son autel qu'Athéna parvient à arracher le suffrage du dieu. Au-delà de la démonstration du sens politique consommé de la déesse, cet échange de bons procédés constitue sans doute également un récit étiologique alternatif aux Dipolies-Bouphonies : la situation du sacrifice dans le temps (le premier) comme dans l'espace (sur l'autel du Polieus) invite à y reconnaitre cette fête. Contrairement au mythe de Sôpatros et consorts, la signification politique est ici on ne peut plus claire : ce Zeus de l'Acropole reçoit un bœuf parce qu'il a permis à sa fille de devenir la divinité tutélaire de la cité. L'élaboration de cette version est malheureusement indatable - même si le rôle attribué au psèphos (jeton de vote autant que suffrage) renverrait plutôt à la période classique - ce qui ne permet pas de la situer par rapport à celle que transmettent les autres sources (parmi celles qui sont datables, la plus ancienne serait Androtion).

Du reste, ces deux récits ont peut-être coexisté et correspondu chacun à une composante de la fête, même s'ils renvoient sans doute au même sacrifice. Au demeurant, bien que cette notice ne fasse que très peu référence au déroulement du rituel, elle explicite bien la signification politique des Dipolies-Bouphonies : c'est aussi la validation de la fonction tutélaire d'Athéna par le vote des dieux et / au nom de la cité qu'elles célèbrent. Ceci renforcerait l'hypothèse que la prêtresse rétribuée à l'occasion de la fête était bien celle de la déesse. Dans ce processus de désignation de la divinité tutélaire des Athéniens, Zeus aurait donc joué un rôle politique en tant que Polieus. Il faut toutefois bien prendre la mesure de celui-ci : dans cette affaire, le dieu reste en arrière-plan. En donnant son suffrage à sa fille, il transmet autant qu'il s'efface, il confère une légitimité tout en se «contentant» du premier sacrifice. Ce récit offrirait un complément à celui des Euménides d'Eschyle, en inversant la perspective : Athéna y vote en faveur d'Oreste parce qu'il a suivi les prescriptions oraculaires d'Apollon; celles-ci étant elles-mêmes l'expression de la volonté de Zeus, la déesse peut justifier son suffrage par le célèbre « je suis toute du côté du père (karta d'eimi tou patros) ${ }^{52}$. Dans la notice d'Hésychios, c'est Zeus qui est du côté de sa fille.

En quittant la logique narrative et dynamique du récit étiologique pour examiner la structure du panthéon (y compris dans l'espace), on peut décrire ce même rapport en des 
termes opposés : dans la sphère politique - sur l'Acropole du moins - Athéna prend autant de place que possible, réduisant Zeus à un rôle mineur. Si le Polieus est politique, il délègue entièrement le rôle tutélaire à sa fille ; et même sous cette acception, on ne peut comprendre l'épiclèse en faisant abstraction de son sens topographique. Comme l'Hupatos et l'Herkeios, le Polieus occupe sur l'Acropole une position de soutien vis-à-vis d'Athéna ${ }^{53}$. La glose d'Hésychios met ainsi en exergue d'autres enjeux que ceux que l'on trouve chez Porphyre, enjeux qui ne portent pas tant sur le rituel que sur l'organisation du panthéon de la cité. Dans cette optique, les préoccupations des Athéniens lors des Dipolies n'étaient pas seulement liées au sacrifice animal: la fête avait aussi pour but de confirmer annuellement la position tutélaire d'Athéna et le soutien que Zeus lui apporte en arrièreplan.

\section{Zeus Polieus dans les dèmes}

Zeus Polieus recevait également un culte à l'échelle des dèmes. On en connaît les modalités pour deux d'entre eux, Erchia et Thorikos, grâce à leurs calendriers sacrificiels respectifs 54 .

\section{Erchia}

Deux entrées du calendrier d'Erchia (ca. 375-360) concernent le culte de Zeus Polieus. La première prévoit pour le 12 Métageitniôn l'offrande au dieu, sur l'Acropole, d'un mouton d'une valeur de 12 drachmes dont les démotes consommaient la viande sur place ${ }^{55}$. Chose remarquable, le document répertorie le même jour les trois seuls autres sacrifices offerts par le dème en astei, à savoir un mouton pour Apollon Lukeios, une brebis pour Déméter dans l'Éleusinion, et une autre pour Athéna Polias, sur l'Acropole ${ }^{56}$. Le regroupement de ces quatre offrandes sur une même journée répond certainement à des raisons pratiques : il devait faciliter la participation des Erchiens ne vivant pas en ville. Son articulation avec le calendrier cultuel n'en reste pas moins problématique, puisque les dates précises des fêtes qui prenaient place en Métageitniôn ne sont pas connues: on ne peut donc déterminer si le 12 de ce mois correspondait à une fête célébrée pour l'une des quatre divinités ${ }^{57}$ ou à un jour proche de chacune d'entre elles ${ }^{58}$. Dans la seconde hypothèse, il serait toutefois difficile de rapprocher les sacrifices au Polieus et à la Polias des Dipolies, célébrées près de deux mois auparavant. Trois hypothèses restent alors envisageables : 1) les Métageitnia ; 2) une fête inconnue en l'honneur des deux divinités en Métageitniôn sur l'Acropole ; 3) les Panathénées, tenues à la fin du mois précédent : il s'agirait alors de la seule indication de la présence du Polieus à cette fête. Quoi qu'il en soit, le calendrier d'Erchia permet d'établir une association du dieu avec sa fille qui n'apparaît qu'en filigrane dans le cadre des Dipolies: dans ce cas, en effet, les démotes leur offrent un sacrifice le même jour, au même endroit.

Les Erchiens consacraient au Polieus un autre mouton d'une valeur identique le 3 Skirophoriôn sur leur propre acropole, sans emporter la viande ${ }^{59}$. Le cadre de cette offrande inviterait à confirmer l'acception topographique de l'épiclèse : le dieu est ainsi surnommé parce qu'on lui rend un culte en (akro)polei, dans le centre urbain comme dans les dèmes. De plus, le calendrier d'Erchia enregistre d'autres sacrifices devant être accomplis le même jour et au même endroit pour Athéna Polias, Aglauros, Poséidon et une autre divinité dont le nom a été effacé, mais qui est très probablement Pandrosos ${ }^{60}$. La 
logique qui fonde cet ensemble laisse peu de place au doute, puisqu'il regroupe des puissances qui ont toutes l'Acropole pour principal lieu de culte. Ce 3 Skirophoriôn, très probablement lors d'une fête commune, les démotes recomposaient donc le cœur des divinités acropolitaines de la citéb ${ }^{61}$ de ce point de vue, l'acropole d'Erchia pourrait être décrite comme une "butte témoin" par rapport à celle d'Athènes ${ }^{62}$. L'intégration du Polieus à ce groupe invite évidemment à considérer le dieu comme politique : il est en effet associé à des dieux qui le sont indubitablement, la Polias en tête; de plus, le dédoublement de son sacrifice athénien à l'échelle du dème constitue un facteur d'homogénéité entre les différents niveaux de la citée ${ }^{3}$. Cependant, une fois de plus, cette coloration fonctionnelle de l'épiclèse n'efface jamais totalement son acception topographique.

En dépit des incertitudes qui subsistent sur le plan héortologique, ces deux sacrifices des Erchiens au Polieus soulignent clairement trois facettes du dieu. Ils confirment son ancrage sur les acropoles, tant celle d'Athènes que celle d'Erchia. Par là-même, ils favorisent l'articulation entre les identités aux échelles politique et démotique: l'interdiction d'emporter les viandes pourrait être le signe d'un souci de favoriser la cohésion des démotes-citoyens, de même que les rites du dème seraient comme une reproduction en miniature de ceux de la cité, du moins en ce qui concerne l'identité des destinataires divins. On remarque enfin que le dieu est directement associé à la Polias, d'une façon qu'on ne rencontre jamais aussi explicitement dans le cadre du culte de la cité.

\section{Thorikos}

Il en va différemment à Thorikos. Le sacrifice adressé au Polieus enregistré dans le calendrier de ce dème présente en effet des variations sensibles par rapport à celui $\mathrm{d}^{\prime}$ Erchia, pourtant à peu près contemporain ${ }^{64}$ :

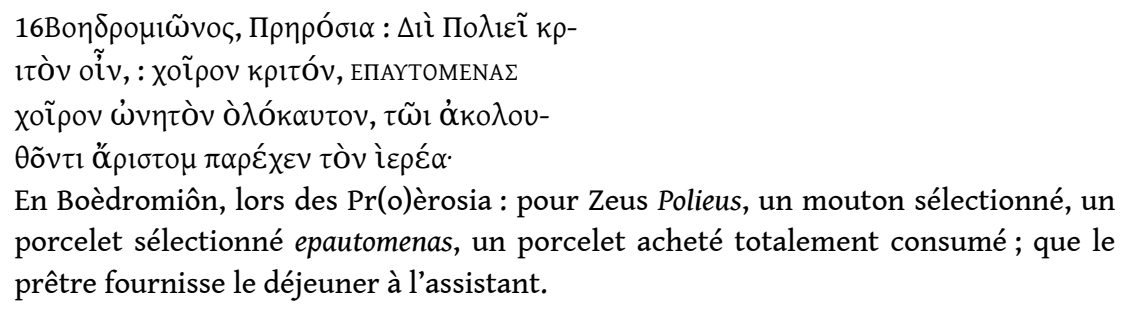

Plus que les aspects organisationnels, sans réelle incidence sur le sens à donner à

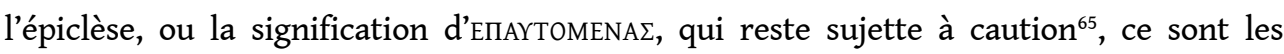
offrandes et surtout l'occasion du sacrifice qui retiennent l'attention. Celui-ci prenait place dans un cadre différent de ceux effectués à Athènes ou à Erchia, puisqu'il avait lieu en Boèdromiôn, lors des Proèrosia : cette fête d'« avant le labour " était célébrée dans plusieurs dèmes, le plus souvent pour Déméter, mais aussi pour Zeus, comme à Myrrhinonte ou, comme ici, à Thorikos. Au-delà des différences de calendrier avec d'autres lieux de l'Attique ${ }^{66}$, ce sont ses finalités qui éveillent la curiosité : si on ne les connaît pas précisément, le nom de cette célébration suggère un lien avec cette étape du travail de la terre. Le choix du Polieus comme récipiendaire des sacrifices invite évidemment à établir un parallèle avec les Dipolies-Bouphonies : même si aucune relation explicite n'est attestée entre elles, ces deux fêtes constituent chacune une réponse à un souci commun (le labour) au moyen d'un sacrifice à un même dieu (le Polieus). Des différences restent toutefois observables: si l'on en croit Porphyre, les Dipolies- 
Bouphonies mettaient en exergue cette préoccupation de la nécessité du labour par le biais d'une mise en scène (l'effigie de la bête bourrée de paille) ; rien n'indique que les Proèrosia de Thorikos aient comporté un rite semblable qui ne pouvait être totalement identique, puisqu'aucun bœuf n'y était sacrifiée ${ }^{67}$. Les réponses rituelles à cette problématique commune qu'apportent les deux fêtes diffèrent également en ce qu'elles interviennent à une étape distincte du calendrier agricole, à savoir à son début et à son terme : la mise à mort d'un bœuf était potentiellement porteuse de sens dans le cadre d'une fête de dissolution / résolution de fin d'année telle que les Dipolies et Bouphonies ; elle l'était peut-être moins dans celui d'un rite préliminaire au redémarrage du cycle agricole qui passe précisément par le travail de l'animal.

Il faudrait donc envisager que le Polieus, celui des Bouphonies athéniennes et des Proèrosia de Thorikos, ait été aussi perçu comme compétent dans le domaine du labour ${ }^{68}$. Du reste, il est possible de mettre en lumière cette compétence du Polieus de Thorikos sans mettre à contribution de façon excessive un parallèle avec les Bouphonies qui a ses limites, on l'a vu. Les Proèrosia avaient lieu en Boèdromiôn, autrement dit à la fin de l'été ou au début de l'automne, une période à laquelle la terre peut être encore très dure en contexte méditerranéen, du fait de la sécheresse estivale. Le travail du laboureur en pâtit, qui doit attendre avec impatience les premières pluies qui permettent d'attendrir la terre. Or, l'eau du ciel est une compétence jovienne entre toutes, ce qui est bien attesté en plusieurs lieux de l'Attique, et en particulier à Thorikos : c'est probablement de ce dème que provient la borne de sanctuaire de Zeus Auantèr, "Assécheur ", qui laisserait penser que l'endroit souffrait d'une pluviométrie faible et surtout très irrégulière, caractéristique de la région athénienne ${ }^{69}$. C'est donc peut-être en tant que faiseur de pluie que le Polieus était invoqué lors de ces fêtes du labour.

Si cette interprétation devait s'avérer exacte, la situation du Polieus de Thorikos au sein du réseau jovien attique serait peut-être plus éloignée des pôles politique et acropolitain que celle du Polieus d'Erchia, et plus proche des pôles élevé, montagnard et

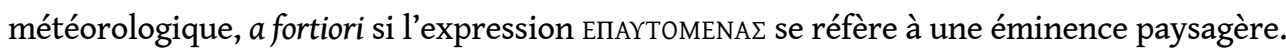
De ce point de vue, l'holocauste du porcelet lors des Proèrosia constituerait un exemple supplémentaire de sacrifice avec combustion complète pour un Zeus élevé. Toutefois, bien que la récurrence semble patente, il serait sans doute audacieux de l'ériger en paradigme sacrificiel et d'en tirer une interprétation univoque ${ }^{70}$.

\section{Du positionnement du Polieus au sein du polythéisme attique}

L'examen de l'ensemble des données relatives au culte de Zeus Polieus en Attique, permet de dresser un tableau du dieu sensiblement plus complexe que celui qui se fonderait sur la seule étude des Bouphonies. La figure du Polieus athénien semble ainsi combiner trois dimensions qui sont mises en exergue avec plus ou moins de netteté selon les contextes dans lesquels il est invoqué.

La première de ces dimensions est spatiale : le Polieus est un dieu de la polis entendue dans son sens topographique. On le retrouve en effet systématiquement sur les acropoles à Athènes et à Erchia. Seuls deux documents invitent à nuancer ce constat : le calendrier de Thorikos et la borne du Cynosarges. En ce qui concerne le premier, la localisation des

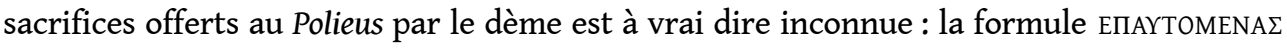


reste énigmatique et il n'est pas assuré qu'elle ait indiqué un lieu. En revanche, on a vu qu'il était possible que l'abaton que délimitait la seconde dépendît du sanctuaire de l'Acropole, quelle qu'ait été sa fonction précise.

La deuxième dimension met en exergue le caractère élevé du Polieus, qui a pu être mis à contribution dans le domaine agricole en tant que faiseur de pluie : en Attique, ce trait est particulièrement caractéristique des Zeus des sommets, pour la plupart compétents en matière de météorologie. Il n'est pas impossible que le dieu ait tenu ce rôle à Thorikos, on l'a vu. C'est, il faut l'admettre, moins net dans le cadre des Dipolies et Bouphonies : si le récit étiologique de Porphyre fait état d'une sécheresse, cette composante se retrouve dans le schéma narratif de tant de mythes qu'elle n'est peut-être pas spécifiquement liée à l'identité du dieu. La dimension agricole du Zeus de l'Acropole n'apparaît que par petites touches; elle pourrait n'être qu'une composante secondaire de son champ de compétence : le Polieus ferait dans ce domaine plutôt figure de pivot au sein du réseau des figures joviennes attiques, en synthétisant, entre autres éléments, la pluviométrie et la question du labour, champs dans lesquels d'autres Zeus sont plus explicitement impliqués.

La troisième dimension du Polieus athénien est politique en ce qu'il est aussi un dieu de la polis-cité ${ }^{71}$. Il l'est dans le sens où il contribue à la cohésion de la communauté politique, en permettant notamment l'articulation entre les identités citoyennes aux échelles politique et démotique. Les sacrifices qu'il recevait des démotes d'Erchia le montrent bien ; de façon moins nette, les Dipolies invitent à le penser également.

Il ne faut toutefois pas se méprendre sur le rôle qui a pu être le sien dans ce domaine et ne pas étendre cette dimension unificatrice jusqu'à un rôle tutélaire complet. De fait, dans la documentation attique, il n'est jamais clairement invoqué en tant que protecteur actif ou étendard de la cité. Seul, cet argument a silentio n'est certes pas suffisant ; il prend en revanche davantage de poids si on le met en parallèle avec les témoignages relatifs à deux autres Zeus qui, à Athènes, semblent avoir été plus clairement impliqués dans la protection active de la communauté politique : l'olumpios et le Sôtèr.

Le premier semble avoir été invoqué de façon privilégiée par les Athéniens dans le cadre de leur politique extérieure: c'est peut-être lui qui percevait la dîme des amendes infligées aux contrevenants du décret de 446/5 organisant la répression de la révolte de Chalcis $^{72}$; dans le traité d'alliance entre Athènes, l'Arcadie, l'Achaïe, Élis et Phlious, conclu peu après la bataille de Mantinée, il est le premier récipiendaire, aux côtés d'Athéna Polias, Déméter et Korè, les Douze dieux et les Semnai theai, d'un sacrifice et d'une procession promis par les Athéniens pour que l'issue des évènements leur soit favorable $e^{73}$.

S'il est un Zeus qui a été invoqué par les Athéniens en vue de la survie de la communauté politique et de la protection de son intégrité, notamment vis-à-vis des menaces extérieures, c'est bien le Sôtèr, du moins à partir $\mathrm{du}_{\mathrm{IV}}^{\mathrm{e}} \mathrm{s}$. - surtout après 338 - et durant l'époque hellénistique. Les témoignages abondent en ce sens, et il n'est pas possible de les citer tous ici ; on en retiendra deux. Dans le Contre Léocrate de Lycurgue, parmi les dieux qui auraient fait les frais de l'impiété de l'accusé, c'est en effet le Sôtèr que l'Orateur distingue : c'est le seul qu'il évoque à plusieurs reprises, ce qui ne laisse pas de doute sur la connotation politique dont il investit cette figure divine, dans un discours irrigué par la notion de sôtèria et prononcé dans le contexte de l'après-Chéronée ${ }^{74}$. Un siècle après, à la suite de la libération du joug macédonien en 229 et jusqu'au début du $\mathrm{I}^{\mathrm{er}} \mathrm{s}$., c'est encore ce 
Zeus qui a les faveurs des soldats athéniens affectés à la garnison de la forteresse de Rhamnonte $\mathrm{e}^{75}$.

41 Ces rôles protecteurs de l'Olumpios et du Sôtèr semblent s'être couplés, à certaines périodes, avec un engouement, un investissement dont on n'a pas de trace aussi nette pour le Polieus. Bien qu'ils soient souvent fragmentaires et partiels, et qu'ils ne permettent pas de dresser un tableau d'ensemble en la matière, les comptes et inventaires de biens sacrés des $\mathrm{V}^{\mathrm{e}}$ et $\mathrm{IV}^{\mathrm{e}}$ siècles peuvent néanmoins donner quelques indications sur la richesse des dieux des Athéniens. Sur ce plan, on l'a vu, les données sont maigres en ce qui concerne le Polieus : on ne sait pas si ce dernier était significativement riche à cette époque, mais il l'était probablement moins que l'olumpios. Ce dernier faisait l'objet de cinq entrées dans les comptes des trésoriers des autres dieux de $429 / 8$, soit plus que tout autre divinité dans ce document ${ }^{76}$ et il figurait encore un siècle plus tard parmi les grands propriétaires divins ${ }^{77}$. Bien entendu, la richesse d'un dieu n'est pas systématiquement révélatrice de son importance théologique et encore moins d'une orientation particulière de son rôle (tutélaire par exemple), mais elle peut en constituer une indication parmi d'autres ${ }^{78}$.

42 Sur ce plan, le nombre de bêtes que la communauté consent à sacrifier à une divinité à un moment donné est peut-être plus directement significatif de l'importance de la protection qui en est attendue : en l'occurrence, on peut juger modeste le total de six bovins répertoriés dans le calendrier de Nikomachos offerts par la cité lors des Dipolies au regard des dizaines d'animaux sacrifiés aux Olumpieia athéniens et surtout aux centaines que reçoit annuellement Zeus Sôtèr dans les années 330, d'après l'estimation que l'on peut tirer des comptes du dermatikon ${ }^{79}$. Il faut certes bien mesurer une telle comparaison: le premier de ces deux documents ne consigne qu'une partie des bêtes sacrifiées, omettant peut-être celles fournies par les dèmes, alors que le second est l'expression d'un total. Néanmoins, cet écart reste probablement significatif de l'importance respective du rôle de ces trois Zeus dans la protection de la cité.

Un dernier indicateur à ce sujet peut être fourni par la fréquence des invocations et des prises à témoin dans la littérature, et notamment celle qui retranscrit la parole publique. Il est toutefois moins probant que les précédents : chez les orateurs, c'est de loin le nom de Zeus « tout court » qui est le plus souvent prononcé. On note cependant que si ceux de Zeus Olumpios ${ }^{80}$ et Zeus Sôtèr ${ }^{81}$ apparaissent tout de même à quelques (rares) reprises, celui de Zeus Polieus en est totalement absent. De même, Zeus n'est jamais désigné comme poliouchos dans la documentation athénienne. Cette appellation est pourtant fréquemment utilisée dans les textes versifiés comme équivalent poétique de Polias / Polieus, mais aussi parfois dans les textes en prose; dans les deux cas, elle a une signification plus spécifiquement fonctionnelle et désigne des divinités - Athéna en premier lieu - qui, littéralement, «tiennent la polis », autrement dit qui "protègent la cité $\aleph^{82}$. Or Zeus poliouchos brille par son absence dans le théâtre et chez les orateurs attiques, en particulier dans l'œuvre d'Eschyle qui se caractérise pourtant par un certain "diocentrisme $»^{83}:$ le Tragique attribue l'épithète à des collectivités de theoi ${ }^{84}$, mais jamais à Zeus. La seule réelle exception se rencontre dans les Lois de Platon ${ }^{85}$, mais le caractère programmatique de ce texte invite à ne pas le tenir pour expressément représentatif de l'environnement religieux des contemporains du philosophe ${ }^{86}$.

D'une façon plus générale, le fait que l'olumpios et le sôtèr aient tenu ce rôle de protecteurs de la cité semble être allé de pair avec leur plus grande perméabilité aux aléas de l'histoire d'Athènes, par comparaison au Polieus. L'exploitation de la figure de l' 
Olumpios par les Pisitratides et par Hadrien est un fait bien connu, sur lequel il n'est pas nécessaire d'insister. L'irruption spectaculaire du Sôtèr dans la documentation politique au IV ${ }^{\mathrm{e}}$ siècle n'est pas moins notable : ce Zeus semble en effet avoir acquis une position de premier plan à partir de cette période, et encore davantage après 338, position qu'il a conservée à l'époque hellénistique (durant laquelle l'olumpios semble d'ailleurs se faire plus discret), avant de rentrer dans le rang sous le Haut-Empire. Contrairement à eux, le Polieus semble avoir conservé sur le temps long une position éminente, stable, mais sans avoir jamais été prééminent dans le panthéon athénien, ni n'avoir été explicitement mis à contribution dans le cadre de la politique ${ }^{87}$. Les places respectives des prêtres de ces trois Zeus au théâtre de Dionysos reflètent sans doute cette histoire : celui du Polieus a conservé la même, une des meilleures, depuis le II ${ }^{\mathrm{e}}$ siècle et durant le Haut-Empire, alors que celui de l'Olumpios n'en a obtenu de comparable qu'au $\mathrm{II}^{\mathrm{e}}$ siècle de notre ère et que le siège attribué à celui du Sôtèr à l'époque impériale est moins bien placé ${ }^{88}$.

Cette absence de rôle tutélaire actif du Polieus à Athènes s'explique par sa proximité, ne serait-ce que topographique, avec la Polias qui a, elle, incontestablement occupé cette fonction. Cette proximité ne prend toutefois pas la forme d'une association directe et systématique. En effet, ce binôme ne fonctionne pas comme les autres couples ZeusAthéna construits autour d'une épiclèse commune en Attique (Boulaios-Boulaia, PhratriosPhratria, Sôtèr-Sôteira, Horios-Horia), où Zeus est toujours l'élément dominant: il est toujours nommé avant Athéna (qui est parfois une Athéna "tout court») et apparaît parfois seul, alors que l'inverse n'est jamais attesté : il n'y a pas d'Athéna Boulaia sans Zeus Boulaios, par exemple.

Le cas du Polieus et de la Polias est fort différent. Les deux divinités n'apparaissent jamais vraiment clairement associées dans les sources, sauf à Erchia: la présence de la déesse aux Dipolies est pour le moins discrète et, à l'inverse, on n'a pas de témoignage assuré de celle du Polieus aux Panathénées. Dans ce cas, le rapport de force entre le dieu et la déesse est totalement déséquilibré et ne peut être en faveur du dieu ; peut-être est-ce la raison pour laquelle ce couple potentiel n'a pas été « activé »: si l'on tient l'ordre d'énonciation pour signifiant, la formule Zeus Polieus kai Athèna Polias n'est pas concevable à Athènes ${ }^{89}$. Les contacts entre ces deux divinités prennent par conséquent une configuration différente de celle que l'on retrouve, par exemple, à la Boulè ou dans les phratries, où l'emploi quasi systématique de l'épiclèse commune « soude » le couple divin. C'est que la Polias est entourée de plusieurs Zeus sur l'Acropole; et d'un point de vue strictement topographique, l'Herkeios et l'Hupatos sont même plus proches de la déesse que le Polieus.

Pourtant, si Athéna Polias ne partage pas son rôle tutélaire avec le Polieus, il y a bien une raison "théologique » au fait qu'ils portent la même épiclèse. Bien sûr, ils sont tous les deux installés sur l'(Akro)polis, mais cette proximité spatiale ne suffit pas à expliquer leur positionnement panthéonique l'un par rapport à l'autre. La logique de ce positionnement réciproque, c'est de l'étiologie alternative des Bouphonies rapportée par la glose d'Hésychios qu'en émane l'expression la plus claire : Zeus délègue le pouvoir à sa fille en échange du sacrifice du premier bœuf. En d'autres termes, il est, en tant que Polieus le garant de la qualité de Polias de sa fille : sa présence pérenne est nécessaire pour soutenir et légitimer la fonction tutélaire d'Athéna. Mais celle-ci l'exerce avec une telle latitude qu'il s'efface dans ce domaine : c'est pourquoi il n'est pour ainsi dire jamais convoqué comme puissance active. Il reste en arrière-plan.

48 Acropolitain, le Polieus l'est indéniablement; il y est fixé, figé même. C'est depuis l' (Akro)polis qu'il garantit la cohésion de la polis autant que la qualité de Polias de sa fille, 
mais ne semble pas occuper de rôle de protecteur actif. Et si sa présence au-dessus de l'épaule d'Athéna est nécessaire, c'est peut-être que sa figure rappelle, synthétise même, celle des Zeus montagnards qui "sur-veillaient" déjà les communautés attiques à l'époque archaïque. Ceux-ci constituent en effet l'arrière-plan paysager et théologique d'une Athènes qui a certes l'Acropole pour centre, mais dont l'horizon est barré par les sommets de l'Hymette ou du Parnès.

\section{Abréviations}

\begin{tabular}{|c|c|}
\hline Agora 18 & $\begin{array}{l}\text { D.J. GEAGAN, The Athenian Agora. XVIII. Inscriptions. The Dedicatory Monuments, } \\
\text { Princeton, 2011. }\end{array}$ \\
\hline Agora 19 & $\begin{array}{l}\text { G.V. LALONDE, M.K. LANGDON, M.B. WALBANK, The Athenian Agora. XIX. Inscriptions. } \\
\text { Horoi, Poletai, Leases of Public Lands, Princeton, } 1991 .\end{array}$ \\
\hline BDEG & $\begin{array}{l}\text { Banque de Données des Épiclèses Grecques (BDEG) du LAHM (UMR } 6566 \text { CReAAH, } \\
\text { Rennes) consultable en ligne : https://epiclesesgrecques.univ-rennes1.fr. }\end{array}$ \\
\hline DELG & $\begin{array}{l}\text { P. CHANTRAINE, Dictionnaire étymologique de la langue grecque. Histoire des mots, Paris, } \\
1968,1999^{2} \text {. }\end{array}$ \\
\hline I.Eleusis & $\begin{array}{l}\text { K. CuINTON, Eleusis. The Inscriptions on Stone. Documents of the Sanctuary of the two } \\
\text { Goddesses and Public Documents of the Deme. I. Text \& Plates (BAAH 236). II. } \\
\text { Commentary (BAAH, 259), Athènes, 2005-2008. }\end{array}$ \\
\hline IGSK 26 & $\begin{array}{l}\text { E. SCHWERTHEIM, Die Inschriften von Kyzikos und Umgebung. Teil II. Miletupolis. } \\
\text { Inschriften und Denkmäler (Inschriften griechischer Städte aus Kleinasien, 26), Bonn, } \\
1983 .\end{array}$ \\
\hline LSCG & Fr. SOKоLOWSKI, Lois Sacrées des cités grecques, Paris, 1969. \\
\hline LSS & Fr. SокоLоWSкI, Lois Sacrées des cités grecques. Supplément, Paris, 1962. \\
\hline NGSL & E. LUPU, Greek Sacred Law. A Collection of New Documents (NGSL), Leiden-Boston, 2005. \\
\hline$P \alpha \mu \nu \circ \tilde{v} \nu \tau \circ \varsigma \mathrm{II}$ & 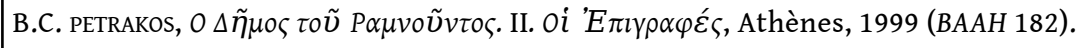 \\
\hline
\end{tabular}

\section{BIBLIOGRAPHIE}

C. BONNET, V. PIRENNE-DELFORGE, « Les dieux et la cité. Représentations des divinités tutélaires entre Grèce et Phénicie », in N. ZENZEN, T. HÖLSCHER, K. TRAMPEDACH (éd.), Aneignung und Abgrenzung. Wechselnde Perspektiven auf die Antithese von "Ost" und "West" in der griechischen Antike, Heidelberg, 2013, p. 201-228. 
U. BRACKERTZ, Zum Problem der Schutzgottheiten griechischer Städte, FUBerlin, Diss., 1976.

A. BRESSON, L'économie de la Grèce des cités. I. Les structures et la production, Paris, 2007.

P. BRULÉ, La fille d'Athènes. La religion des filles à Athènes à l'époque classique. Mythes, cultes et société, Paris, 1987.

P. BRULÉ, Comment percevoir le sanctuaire grec ? Une analyse sensorielle du paysage sacré, Paris, 2012.

W. BURKERT, Homo Necans. Rites sacrificiels et mythes de la Grèce ancienne, Berlin, 1972, 1997², (trad.

fr. H. Feydy, Paris, 2005).

W. BURKERT, La religion grecque à l'époque archaïque, Stuttgart, 1977, 2010² (trad. fr. P. Bonnechere, Paris, 2011).

S.G. BYRNE, Roman Citizens of Athens, Leuven, 2003.

S.G. COLE, « Civic Cult and Civic Identity », M.H. HANSEN (éd.), Source for the Ancient Greek City-State. Symposium August 24-27, 1994. Acts of the Copenhagen Polis Center vol. 2, Copenhague, 1995, p. 292325.

А.В. соок, Zeus. A Study in Ancient Religion. III. Zeus God of the Dark Sky (Earthquakes, Clouds, Wind, Dew, Rain, Meteorites), Cambridge, 1940.

G. DAUX, « Chronique des fouilles et découvertes archéologiques en Grèce en 1959 », BCH 84

(1960), p. 617-874.

G. DAUX, « La grande démarchie : un nouveau calendrier sacrificiel d'Attique (Erchia) », BCH 87 (1963), p. 603-634.

G. DAUX, « Le calendrier sacrificiel de Thorikos au musée J. Paul Getty », AC 52 (1983), p. 150-174.

L. DEUBNER, Attische Feste, Darmstadt, 1932 (réimpr. 1969).

J.-L. DURAND, Sacrifice et labour en Grèce ancienne, Paris-Rome, 1986.

Th. eliopoulos, « Athens : News from the Kynosarges Site », H. FRIELINGHAUs, J. STROSZECK (éd.), Neue Forschungen zu griechischen Städten und Heiligtümern. Festschrift für Burkhardt Wesenberg zum 65. Geburtstag, Möhnesee, 2010, p. 85-91.

St. GEORGOUDI, «L' "occultation de la violence" dans le sacrifice grec : données anciennes, discours modernes ", in St. GEORGOUDI, R. косн PIETTRE, Fr. SCHMIDT (éd.), La cuisine et l'autel. Les sacrifices en questions dans les sociétés de la Méditerranée ancienne, Turnhout, 2005, p. 115-147.

St. GEORGOUDI, « Le consentement de la victime sacrificielle : une question ouverte », in V. MEHL, P. BRULÉ (éd.), Le sacrifice antique. Vestiges, procédures et stratégies, Rennes, 2008, p. 139-153.

B. HoltzmanN, L'Acropole d'Athènes, Paris, 2003.

J.M. HURWIT, The Athenian Acropolis. History, Mythology, and Archaeology from the Neolithic Era to the Present, Cambridge, 1999.

P. ISMARD, La cité des réseaux. Athènes et ses associations. $v I^{e}-I^{e r}$ siècle av. J.-C., Paris, 2010.

O. JAHN, Giove Polieo in Atene, Leipzig, 1865.

M.H. JAMESON, « Notes on the Sacrificial Calendar from Erchia », BCH 89 (1965), p. 154-172.

M.H. JAMESON, « Sacrifice and Animal Husbandry in Classical Greece », in C.R. WHITTAKER (éd.), Pastoral Economies in Classical Antiquity, Cambridge, 1988, p. 87-119.

St. D. LAMBERT, The Phratries of Attica, Ann Arbor, 1993. 
St. D. LAMBERT, « The Sacrificial Calendar of Athens », ABSA 97 (2002), p. 353-399.

M.K. LANGDON, A Sanctuary of Zeus on Mount Hymettos, Princeton, 1976 (Hesperia, suppl. 16).

C. LECOMTE, « L'Athéna de Platon », Kernos 6 (1993), p. 225-243.

M. MAASS, Die Prohedrie des Dionysostheaters in Athen, Munich, 1972.

V. MEHL, « Au plus près de l'autel, la circumambulation au cours des sacrifices », REA 104 (2002),

p. 25-48.

B.D. MERITT, « Greek Inscriptions », Hesperia 37 (1968), p. 266-298.

K. MEULI, « Griechische Opferbräuche », in O. GIGON, Phyllobolia für Peter von der Mühll zum 60. Geburtstag am 1. August 1945, Bâle, 1946, p. 185-288.

J.D. MIKALSON, Honor Thy Gods. Popular Religion in Greek Tragedy, Chapel Hill-Londres, 1991.

J. NEILS, « Athena, Alter ego of Zeus », in S. DEACY, A. VILLING (éd.), Athena in the Classical World, Leiden-Boston-Cologne, 2001, p. 219-232.

M.P. NILSSON, Griechische Feste von religiöser Bedeutung mit Ausschluss der Attischen, Leipzig, 1906 (rééd. Stuttgart-Leipzig, 1995).

R. OSBORNE, The Classical Landscape with Figures. The Ancient Greek City and its Countryside, Londres, 1987.

R. PARKER, Miasma. Pollution and Purification in Early Greek Religion, Oxford, 1983 (rééd. 1996).

R. PARKER, Athenian Religion. A History, Oxford, 1996.

R. PARKER, Polytheism and Society at Athens, Oxford, 2005.

R. PARKER, «A Festival that Moved, and other Problems relating to Skirophorion », in A.P.

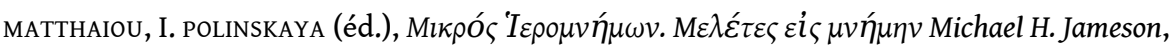
Athènes, 2008, p. 35-39.

St. PAUL, « À propos d'épiclèses “trans-divines” : le cas de Zeus et d'Athéna à Cos ", ARG 12 (2010), p. $65-81$.

St. PAUL, Cultes et sanctuaires de l'île de Cos, Liège, 2013 (Kernos, suppl. 28).

V. PIRENNE-DELFORGE, Retour à la source. Pausanias et la religion grecque, Liège, 2008 (Kernos, suppl. 20).

P. SCHMITT-PANTEL, La cité au banquet. Histoire des repas publics dans les cités grecques, Rome, 1992 ( Collection de l'École française de Rome, 157).

E. SCHWEIGERT, « Greek Inscriptions », Hesperia 9 (1940), p. 309-357.

Sc. SCULLION, « Olympian and Chthonian », ClAnt 13 (1994), p. 75-119.

Sc. SCULLION, « Three Notes on Attic Sacrificial Calendars », ZPE 121 (1998), p. 116-122.

G.P. STEVENS, « The Northeast Corner of the Parthenon », Hesperia 15 (1946), p. 1-26.

A. TомA, « Epiteti di collettività divine nelle tragedie di Eschilo », in O. vox (éd.), Materiali di nomenclatura divina greca, Lecce, 2008, p. 89-104.

A. WILLIAMS, « Leasing of Sacred Land in $4^{\text {th }}$-century Athens. A Reassessment of Six Inscribed Fragments », Hesperia 80 (2011), p. 261-286. 


\section{NOTES}

1. En guise d'aperçu nécessairement incomplet, on se bornera ici à citer quelques jalons au sein d'une bibliographie devenue pléthorique depuis JAHN (1865) : NILSSON (1906), p. 14-16; DEUBNER (1932) [1969], p. 158-174; СOOK (1940), p. 570-605 ; BURKERT (1972) [2005], p. 180-185; BURKERT (1977) [2011], p. 312-313; DURAND (1986); SCHMITT-PANTEL (1992), p. 139-140; PARKER (2005), p. 187-191; PIRENNE-DELFORGE (2008), p. 236-239.

2. Le fait que de grandes synthèses - par ailleurs excellentes - sur la religion grecque (BURKERT [1977] (2011), p. 185 et 255) et le polythéisme athénien (PARKER [2005], p.397) déduisent simplement la fonction de protecteur de la cité du Polieus du sens littéral de l'épiclèse sans davantage la discuter est sans doute révélateur de la prégnance de cette apparente évidence.

3. Le fait que l'étymologie de $\pi 0 ́ \lambda ı \varsigma$ semble devoir être rattachée à cette dernière signification ( DELG, s.v. ; cf. également Pausanias, I, 26, 6 et le commentaire afférent de BONNET, PIRENNE-DELFORGE [2013], p. 210-211) ne peut être retenu pour le présent propos, qui ne concerne que l'épiclèse qui en dérive. J'écarte d'emblée le sens de «ville » ou d'« agglomération ", également bien attesté, mais qui n'est pas opératoire dans le cas présent.

4. Ce sens est premier selon COLE (1995), p. 302-305. Dans la mesure où le cas est généralisable, le

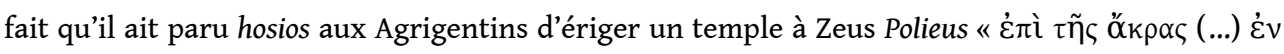

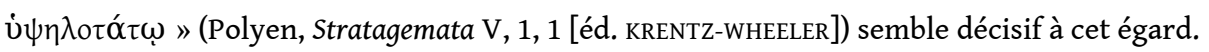

5. Sur la notion de divinité tutélaire, cf. le modèle proposé par BRACKERTZ (1976), p. 155-160, avec les correctifs de COLE (1995) ainsi que les remarques salutaires de PAUL (2013), p. 309-310 et de BONNET, PIRENNE-DELFORGE (2013) : on ne s'étendra plus sur le fait que le cumul de l'épiclèse Polias et de la position tutélaire dont jouit la déesse à Athènes ne saurait constituer une règle générale. 6. J'insiste sur cette précision: d'une cité à l'autre, une figure divine désignée par une même épiclèse n'a pas nécessairement les mêmes fonctions et la même position au sein du panthéon; en l'espèce, c'est le cas pour le Polieus. On se gardera donc bien de généraliser le présent propos à l'ensemble du monde grec, même si l'on pourra tirer grand profit de la mise à contribution d'exemples extra-attiques, précisément dans une optique comparatiste. Cf. notamment les travaux de PAUL (2010) et (2013), p. 270-273 et 313-316, qui étudient notamment le rôle du Polieus et ses liens avec la Polias à Cos, en soulignant leur singularité par rapport aux configurations athénienne et rhodienne de ce couple divin. On notera néanmoins qu'en Attique comme ailleurs, l'épiclèse Polias est attribuée à la seule Athéna, tout comme sa forme masculine est exclusivement jovienne. La seule exception, qu'on ne rencontre qu'en Égypte à l'époque impériale, est ce Sarapis qui est Polieus sans doute par identification à Zeus.

7. Par commodité, il sera ainsi question de culte «politique " ou "à l'échelle politique » pour désigner ce qui concerne la cité d'Athènes dans son ensemble, par distinction de ce qui relève d'associations «infra-politiques" (dèmes, phratries, genè, etc.); bien entendu, celles-ci ne sont pas moins politiques, puisqu'elles concernent les mêmes citoyens. Sur l'articulation entre ces différentes composantes de l'identité citoyenne, cf. ISMARD (2010), à qui j'emprunte donc ce terme d'« associations».

8. Pausanias, I, 24, 4. Le Périégète évoque un agalma sculpté par Léocharès et un autre Zeus qui,

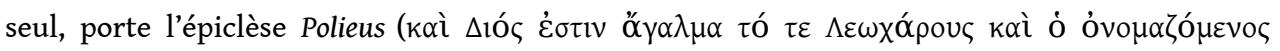
По入ıєú ).

9. Sur le sanctuaire du dieu sur l'Acropole, cf. STEVENS (1946), notamment p. 12-15, qui propose une restitution du plan et de l'élévation du sanctuaire, HURWIT (1999), p. 190-192 et HOLTZMANN (2003), p. 32-33, où l'on trouvera le plan mis à jour par M. Korrès (Fig. 6, no 20) : ce dernier 
intègre notamment l'hypothèse d'un enclos à ciel ouvert de plus grandes dimensions, qui étendait son emprise jusqu'aux murs de la citadelle au nord et au grand autel d'Athéna à l'ouest.

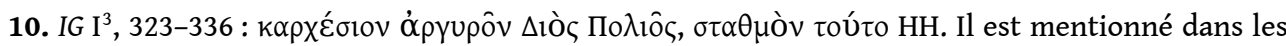
inventaires suivants: IG I ${ }^{3}, 323$ (428/7), 1.53-54; 324 (427/6), 1.64; 325 (422/1), 1. 8-9; 326 (421/0), 1. 20 ; 327 (420/419), 1. 31-32 ; 328 (419/8), 1. 44-45 ; 329 (418/7), 1. 8-9; 330 (417/6), 1. $23-$ 24 ; 331 (416/5), 1.38-39; 333 (414/3), 1. 7-8; 334 (413/2), 1.21-22; 336 (411, sous les Quatre-

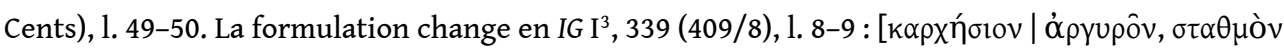

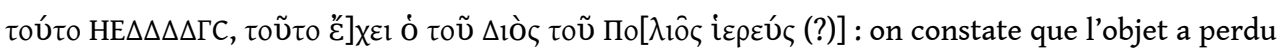
une drachme, peut-être sous l'effet de l'usure ou d'un prélèvement (ce que l'on appellerait le frai en numismatique). Il a en tout cas été repesé, peut-être à la suite du rétablissement de la démocratie après l'épisode des Quatre-Cents; est-ce pour cette raison que le prêtre en a eu la garde ? À partir de 405/4 au plus tard, on retrouve la formulation habituelle, avec le nouveau

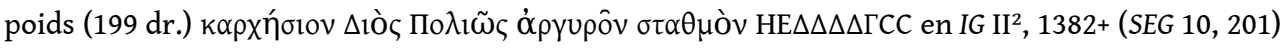
$=I G \mathrm{I}^{3}, 342\left(406 / 5\right.$ ou 405/4), IG II ${ }^{2}, 1388(398 / 7)$ et 1401 (394/3).

11. On ne peut évidemment se faire une idée de l'étendue des biens du dieu à partir du seul karchèsion, pas plus que des comptes des trésoriers des autres dieux pour l'année 429/8 (IG I³, 383) : dans l'entrée qui leur est afférente (1. 139-140), leur montant est en effet effacé.

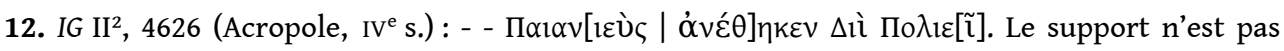
davantage instructif, compte tenu de son état fragmentaire.

13. ELIOPOULOS (2010), p. 88-91 (SEG 57, 70).

14. Contrairement aux abata de Zeus Kataibatès ( $I G \mathrm{II}^{2}, 4964,4965$ et peut-être 4998) qui sont essentiellement définis comme tels parce que le dieu y est « descendu » en les frappant avec la foudre. Cf. PARKER (1983) [1996], p. 167-168 ; BRULÉ (2012), p. 120-123.

15. Cf. les trois cippes définissant le sanctuaire des Tritopatreis comme abaton découverts aux abords du cimetière du Céramique $\left(I G \mathrm{I}^{3}, 1066 ; c a .445-410\right)$. Sur la préservation de la pureté de l'espace sacré, dans le sens le plus prosaïque comme le plus idéel, cf. BRULÉ (2012), passim, et, toujours, PARKER (1983) [1996].

16. Selon Eliopoulos, l'espace délimité par la borne du Cynosarges doit être intégré à l'ensemble des sanctuaires de la région de l'ilissos, dont certains sont des répliques de ceux de l'Acropole. L'hypothèse n'est pas irrecevable en soi, mais l'interprétation de la célèbre description de l'ancienne Athènes par Thucydide (II, 15, 3-4) sur laquelle elle repose est peut-être abusive : l'historien antique ne fait que constater la commune ancienneté des hiera dans ces deux parties de la cité.

17. Au sujet des Dipolies et Bouphonies, je renvoie une fois pour toutes à Androtion, $324 \mathrm{~F} 16$ (éd. JACOBY), Pausanias, I, 24, 4 et 28, 10, Élien, Histoire Variée VIII, 3 (éd. HERCHER) et Porphyre, De Abstinentia II, 10 et 29-30. Pour les compléments, voir la bibliographie afférente supra, n. 1 et la notice de J. Bouffartigue dans l'édition des CUF de Porphyre, De l'abstinence, p. 51-58. Pour l'épigraphie, cf. infra.

18. Sur le faste et l'annualité de la fête, cf. Scholie récente à Aristophane, Nuées, 985 (éd. KOSTER) et, indirectement, Pausanias, I, 28, 10. On trouve la date du 14 Skirophoriôn dans une scholie à

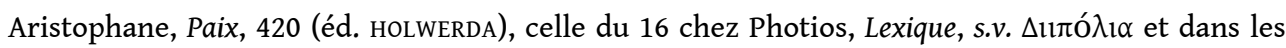
Anecdota Graeca I, s.v. $\Delta$ lıtó $\lambda_{1} \alpha$ (éd. BEKKER, p. 238). La première date tendrait à être confirmée par un règlement athénien $\mathrm{du} \mathrm{IV}^{\mathrm{e}} \mathrm{s}$. ( $\mathrm{cf}$. infra, n. 19) et par un calendrier sacrificiel retrouvé à Miletoupolis, près de Cyzique (IGSK 26, 1, IV ${ }^{\mathrm{e}} \mathrm{s}$.; sur cette inscription et sa possible provenance athénienne, cf. PARKER [2005], p. 484-485).

19. MERITT (1968), p. 267-268, no 3 (SEG 25, 82) = LSCG 179 (Athènes, IV s.), 1.3-10: [- -

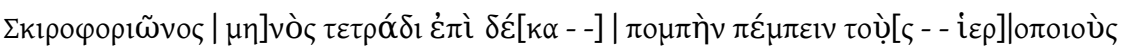

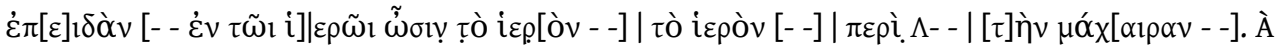


l'exception de sa proposition $\pi \varepsilon p l[\varepsilon] \lambda[\alpha u ́ v \varepsilon ̣ ı v]$ à la ligne 9 , j'écarte les restitutions trop généreuses de Sokolowski.

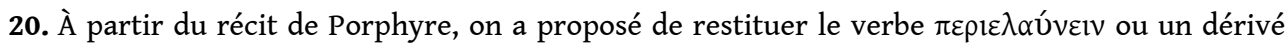
dans le règlement athénien du IV ${ }^{\mathrm{e}} \mathrm{s}$. (cf. supra, n. 19) et dans l'entrée du calendrier de la cité (dit «de Nikomachos») relative aux Dipolies (cf. infra, n. 22). Sur la circumambulation lors des Dipolies-Bouphonies, cf. DURAND (1986), notamment p. 89-94 pour le dossier iconographique. Bien entendu, la pratique n'était pas spécifique à cette fête : pour une mise en perspective, cf. MEHL (2002).

21. Androtion, Élien, les scholiastes et les lexicographes n'évoquent qu'un seul animal et sa mise à mort. Seule une notice isolée (Anecdota Graeca I, s.v. Bovpovía [éd. BEKKER, p. 221]) fait allusion

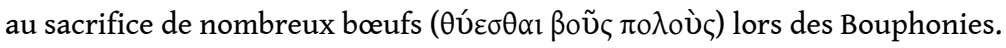

22. LAMBERT (2002) (SEG 52, 48), face B, fr. 1 (IG II ${ }^{2}$, 1357a; LSCG 17 A-B), Col. 2, 1. 7-15

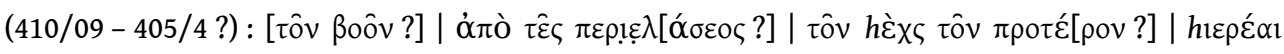

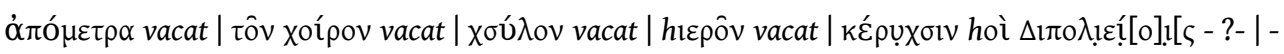
verbe; part ?]. L'entrée évoque également une prêtresse et des hérauts (cf. infra), ainsi que des porcelets et du bois, provisionné pour leur holocauste selon scullion (1994), p. 85. Si ce probable holocauste était bien destiné à Zeus Polieus, il pourrait donner sens à la présence d'une fosse dans la cella du temple de l'Acropole.

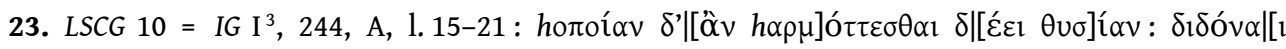

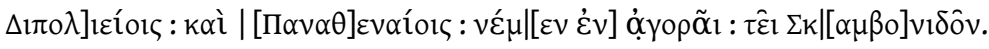

24. IG XII 4, 278. Sur le dossier de Cos, je renvoie à la récente et solide étude de PAUL (2013), notamment p. 30-40 et 261-281 pour ce sacrifice.

25. Et inversement: Élien qualifie le rituel des Dipolies et Bouphonies d'usage typiquement attique (Attikon ethos).

26. La dimension unificatrice, "pan-politique " des Synoikia, qui apparaissent dans la même inscription ( $\left.L S C G 10=I G \mathrm{I}^{3}, 244, \mathrm{C}, 1.16-19\right)$, indiquerait que telle est la couleur d'ensemble du document et, par conséquent, des Dipolies. Sur les Synoikia, cf. PARKER (2005), p. 480-481. Sur la proximité des Panathénées et des Dipolies, voir encore Aristophane, Paix, 418-420.

27. Cf. également Scholie à Homère, Iliade XVIII, 483 (éd. ERBSE) et Souda, s.v. $\Theta \alpha u ́ \lambda \omega v, \Theta \alpha u ́ \lambda \omega v o \zeta$.

28. Androtion, apud Tzetzès à Aristophane, Nuées, 985. Cette citation n'est pas reprise parmi les fragments de l'Atthidographe, ni par Müller ( $F H G)$, ni par Jacoby $(F G r H)$.

29. L'intervention d'un Diomos dans le mythe étiologique des Bouphonies peut laisser entendre que les habitants de Diomeia auraient, à un moment donné, revendiqué un rôle lors de cette cérémonie. La probable localisation de ce dème près du Cynosarges pourrait donner sens au lieu de découverte de la borne de l'abaton de Zeus Polieus. ELIOPOulos (2010), p. 88-91 (cf. SEG 57, 70) y trouve un argument pour supposer que cet abaton ait pu constituer le cadre d'une activité cultuelle propre, distincte des Dipolies et de l'Acropole, activité cultuelle dont les contours héortologiques demeureraient néanmoins flous. Sans aller jusque-là, on peut plus simplement y voir le reflet du rôle particulier de ce dème lors de la fête, qui pourrait être lié au pacage des bœufs dans l'hypothèse où l'abaton aurait eu fonction d'étable sacrée.

30. Sur les daitroi, dont le statut et le rôle lors des Dipolies restent obscurs, cf. PARKER (1996), p. 320-321. Sur les Dipoliastai, nécessairement liés à la fête, compte tenu de leur nom, cf. LAMBERT (1993), p. 363-364 et PARKER (1996), p. 334. Leurs prérogatives sont tout aussi mystérieuses : en Attique, ils ne sont connus que par quelques lignes d'un inventaire du IV eiècle (ca. 330) qui consignent la vente d'un terrain sis à Phlya par deux épimélètes de l'association (sCHWEIGERT [1940], p. 330-332, no 38 [SEG 21, 578] A, 1. 5-7).

31. PARKER (1996), p. 300. Ils étaient peut-être également chargés du probable holocauste de porcelets enregistré dans la même entrée du calendrier (scullion [1994], p. 85).

32. LSCG 179 (SEG 25, 82), 1. 5-7 (cf. supra, n. 19). 
33. PARKER (1996), p.299. Le terme bouphonos apparaît chez Pausanias, celui de boutès chez

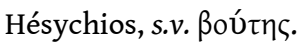

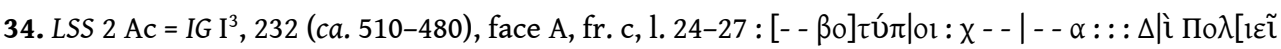

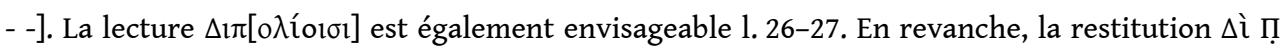

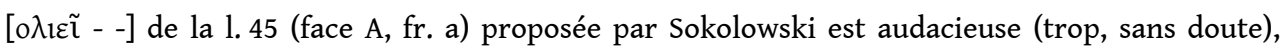
puisque la lecture du pi n'est pas assurée.

35. PARKER (1996), p. 299. L'association de la fonction de boutupos à celle de prêtre de Zeus Teleios sur le siège de proédrie de ce dernier $\left(I G \mathrm{II}^{2}, 5075\right)$, pourrait aller dans ce sens. Toutefois, le terme

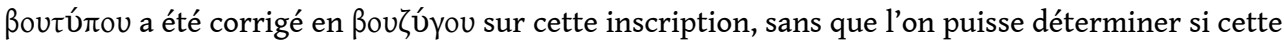
modification procède d'une réorganisation du culte ou d'une erreur du lapicide corrigée ensuite ( MAASs [1972], p. 136-137).

36. Seuls les inventaires de l'Hécatompédon indiquent peut-être qu'il tenait un rôle dans la gestion des biens du dieu à la fin du ve s. (cf. supra, n. 10). Dans une inscription du monument de Sérapion, l'association des différentes fonctions listées avec la prêtrise du Polieus s'explique par un processus de cumul plutôt que par une réelle cohérence sacerdotale, Agora 18, C122

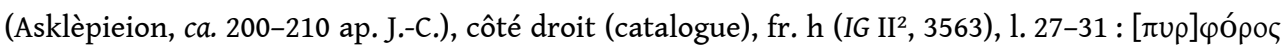

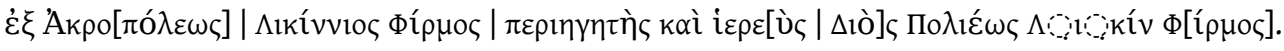
Sur ce Licinnius Phirmos de Gargèttos, cf. BYRNE (2003), p. 343-344.

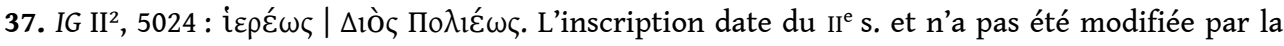
suite (MAASS [1972], p. 104). Sur la localisation du siège, cf. infra, n. 88.

38. Selon Porphyre, De Abstinentia II, 10,2, Diomos était déjà prêtre de Zeus Polieus lorsqu'il égorgea (esphaxe) le premier bœuf.

39. LAMBERT (2002) (SEG 52, 48), face B, fr. 1, Col. 2, 1. 10 (cf. supra, n. 22).

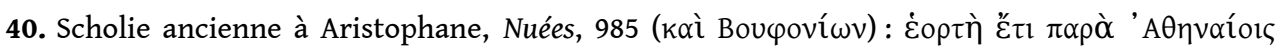

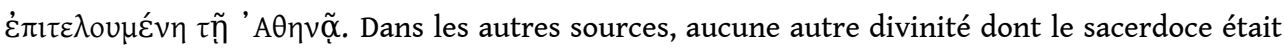
occupé par une femme n'est associée avec les Dipolies ou avec Zeus Polieus.

41. Ou, présenté du point de vue inverse, si les nombreux genè créés et / ou redéfinis comme groupes aristocratiques au cours de l'époque hellénistique (sur ce phénomène, cf. ISMARD [2010], p. 365-404) ont pu trouver dans les Dipolies un terrain à investir et où ils ont éventuellement pu entrer en compétition.

42. BURKERT (1972) [2005], p. 180-185.

43. MEULI (1946), notamment suivi par BURKERT (1972) [2005], p. 180-185, non sans quelques ajustements.

44. Cf. notamment GEORGOUDI (2005), p. 134-138 et (2008), p. 142-146, ainsi que PIRENNE-DELFORGE (2008), p. 236-239.

45. Sur ce point, cf. PARKER (2005), p. 187-191.

46. On a mis au jour des dépôts d'ossements d'animaux brûlés aux sanctuaires archaïques de Zeus sur le Parnès (cf. DAUx [1960], p. 658 et LANGDON [1976], p. 100-101) et l'Hymette (cf. LANGDON [1976], p. 76-77) ce qui laisse supposer que le dieu y recevait des sacrifices avec combustion complète. Sur le Tertre d'Erchia, on offrait l'holocauste d'un porc à Zeus Epôpetès (LSCG 18, Col. III, 1. 20-26).

47. Pausanias, I, 26, 5 (pemmata) et VIII, 2, 3 (pelanoi).

48. PIRENNE-DELFORGE (2008), p. 234-239.

49. On peut donc constater avec scullion (1994), p. 81-90 la pluralité des modes sacrificiels du culte du Polieus, sans nécessairement attribuer, comme lui, une dimension chthonienne à Zeus ou surévaluer son rôle agricole.

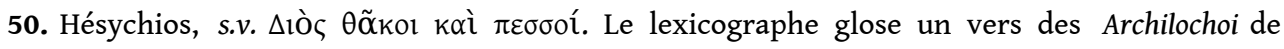
Cratinos (fr. 7, éd. коск). On y trouve une allusion succincte chez Photios, Lexique (= Souda), s.v. $\Delta$ iò $\psi \tilde{\eta} \varphi \circ \varsigma$. 
51. Varron, apud Augustin, Cité de Dieu XVIII, 9 (éd. DOMBART-KALB). Ps-Apollodore, Bibliothèque III, 14, 1 (éd. WAGNER).

52. Eschyle, Euménides, 566-777, et notamment 616-625 et 736-738. Sur l'interprétation de la réplique d'Athéna, cf. NEILS (2001), en particulier p. 219-220.

53. L'autel de l'Hupatos était situé à l'entrée de l'Erechtheion et celui de l'Herkeios, sous l'olivier sacré.

54. On laissera de côté la possible implication du dème de Diomeia dans le culte du Polieus, qui reste incertaine et a fortiori la tradition transmise par Tzetzès qui localise les Bouphonies à Braurôn (qui n'est, du reste, pas un dème).

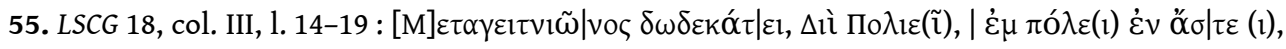
oĩ $\varsigma$, oủ $\varphi$ opló, $\Delta C C$.

56. Ibid., col. I, 1. 2-6 ; col. II, 1.2-6 ; col. IV, 1. 14-18. Un autre sacrifice du calendrier est offert dans l'asty (à Agrai très précisément), celui pour le Meilichios, lors des Diasia ; mais il est distinct de l'ensemble formé par les quatre autres (sur les Diasia, cf. PARKER [2005], p. 466).

57. DAUX (1963), p. 620-621.

58. Le sacrifice à Déméter pourrait être rapproché des Éleusinia, célébrées en Métageitniôn ou au début de Boèdromiôn, et celui adressé à Apollon Lukeios, des Métageitnia (sur ces deux fêtes, cf. PARKER [2005], respectivement p. 468-469 et p. 475).

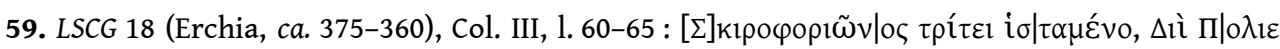

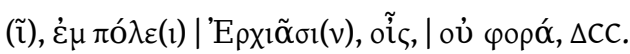

60. Ibid., respectivement Col. I, 1. 58-62 ; Col. II, 1. 56-60 ; col. IV, 1. 57-61 ; col. V, 1. 66-67 (seul le mois n'est pas effacé ; JAMESON [1965], p. 156-158 a le premier reconnu Pandrosos dans la récipiendaire de ce sacrifice).

61. PARKER (2005), p. 68, qui préfère ne pas formuler d'hypothèse au sujet de cette probable fête. JAMESON (1965), p. 156-158 propose d'identifier ces sacrifices avec les arrhéphories, célébrées en Skirophoriôn pour Athéna et Pandrosos (sur cette fête, cf. PARKER [2005], p. 221-222 et 461). Cf. également BRULÉ (1987), p. 35-36.

62. La portée théologique de cette métaphore ne correspond pas nécessairement à une réalité géologique.

63. Le parallèle n'est pas parfait, puisque les bêtes ne sont pas les mêmes à l'échelle de la cité et à celle du dème, ce que l'on relativisera en ce qui concerne le calendrier d'Erchia. Les contraintes matérielles semblent y influer sur le choix des animaux sacrifiés : ils sont en effet peu coûteux dans l'ensemble ; de plus, l'entrée relative à la Polias précise le fait que l'ovin est offert «à la place d'un bœuf » (antibous).

64. NGSL 1, 1. 13-16. La datation de l'inscription reste discutée (ca. 440-430 ? 430-420 ? 380-375 ?). 65. Sur la lecture et la traduction d'ЕПАҮтомЕNA乏, cf. en dernier lieu NGSL 1, p. 132-133, où Lupu,

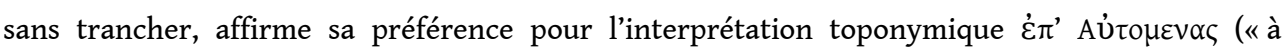
Automena »), peut-être un lieu élevé; le cas échéant, cela mettrait en relief l'acception topographique de l'épiclèse Polieus. Dans l'ed.pr., DAUX (1983) (SEG 33, 147) proposait de

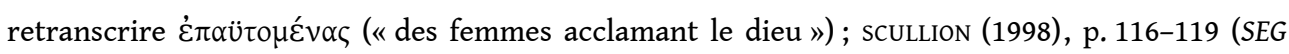

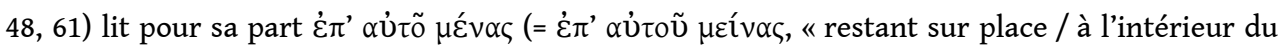
sanctuaire »).

66. Sur les Proèrosia, cf. PARKER (2005), p. 330-332 et 479. La fête était célébrée le 6 Pyanopsiôn à Éleusis.

67. Ce que l'on se gardera de surinterpréter: comme à Erchia, l'absence de sacrifice bovin s'explique sans doute aussi (surtout?) par les moyens limités du dème.

68. Il partagerait ce domaine d'intervention avec d'autres Zeus attiques, comme le Teleios ou celui du Palladion : les prêtrises de ces deux dieux ont été associées à la fonction de bouzyge (cf. e.g. IG 
$\mathrm{II}^{2}, 5055$ et 5075) qui était en charge d'accomplir le labour sacré au Bouzygion, en contrebas de l'Acropole (PARKER [1996], p. 286-287).

69. IG $\mathrm{II}^{2}, 2606$ ( $\mathrm{IV}^{\mathrm{e}} \mathrm{s}$.). Ailleurs en Attique, la compétence de Zeus en matière de pluie est encore soulignée par d'autres épiclèses du dieu telles qu'Ombrios, Apèmios, Sèmios/Sèmaleos, mais aussi Parnèssios, Humèttios... Sur la pluviométrie, inférieure dans cette région à $400 \mathrm{~mm}$ annuels en moyenne et essentiellement concentrée sur les mois d'automne et d'hiver (octobre-mars), cf. OSBORNE (1987), p. 31-34 et BRESSON (2007), p. 41-43.

70. Cf. supra, p. 97 et n. 49.

71. J'écarte ici le champ des institutions politiques: dans ce domaine, le Polieus est absent, s'effaçant derrière des Zeus plus « spécialisés » en la matière, tels que le Boulaios pour la Boulè et, très probablement, l'Agoraios pour l'Ekklesia.

72. IG I ${ }^{3}, 40,1.32-36$. Compte tenu du caractère fortement impérialiste de ce décret, il me semble que c'est bien à l'Olumpios d'Athènes qu'il est fait référence dans ce passage, et non à celui de Chalcis, dans le sanctuaire duquel le texte devait être exposé (Ibid., 1.61-63). On ne peut absolument exclure, toutefois, que la dîme soit versée au Zeus d'olympie - la pratique est fréquente - même si on s'expliquerait mal que les Athéniens aient ressenti le besoin de valider dans le sanctuaire panhellénique leur victoire / répression sur une cité de l'archè, eubéenne qui plus est.

73. IG $\mathrm{II}^{2}, 112$ (362/1), 1.6-12. On ne manquera pas de souligner la première place de l'olumpios dans la liste, ainsi que son association avec Athéna Polias, qui apparaît en deuxième position.

74. Contre Léocrate 17 (avec Athéna Sôteira) et 136-137 (le dieu, seul, est évoqué trois fois, avec un effet d'insistance). En dehors de l'invocation liminaire d'usage à Athéna et aux autres dieux et héros de la cité (Ibid., 1) et quelques références de circonstance à des sanctuaires ou des ancêtres héroïques, les dieux ne sont autrement invoqués dans ce discours que comme une communauté indéfinie de theoi (passim).

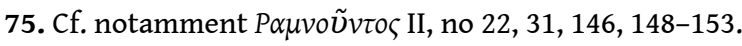

76. IG I ${ }^{3}, 383,1.78-79,269-270,276-277,325-326,348-349$. La plupart des montants étant illisibles, on ne peut pas donner grand sens à celui de la première entrée, le seul qui ait été conservé pour l'olumpios (4931 dr.), faute de pouvoir le comparer à ceux d'autres divinités d'importance similaire (Apollon Puthios e.g.). Pour le Polieus, cf. supra, n. 11.

77. Agora 19, L6 (343/2), 1. 74-75, 77-78, 81, (84 ?), 145 = Williams (2011), stèle III, 1. 1-2, 4-5, 1. 7-8, (11 ?), 26 répertorie plusieurs maisons (oikiai) et un domaine (temenos) appartenant à Zeus Olumpios. Selon Williams, la répartition des biens du dieu sur deux stèles différentes (l'ensemble en aurait compté quatre) serait révélateur de l'étendue de ses richesses. On tempérera ce constat en rappelant que les fragments retrouvés ne conservent qu'une modeste partie du document originel et que d'autres divinités pouvaient se trouver dans ce cas.

78. Dans ce domaine, Athéna Polias écrase évidemment toute concurrence à Athènes : sur la période 433/2-423/2, elle aura prêté près de $4750 \mathrm{~T}$ à la cité, à côté desquels les quelques $820 \mathrm{~T}$ des autres dieux paraissent bien modestes (IG $\left.\mathrm{I}^{3}, 369\right)$.

79. IG $\mathrm{II}^{2}, 1496$, col. IV, 1.82-83 (Olumpieia de 334/3) et 118-119 (Olumpieia de 333/2) : respectivement un peu plus de 291 et au moins 200 dr., soit 96 et au moins 70 bêtes. Ibid., 1. 89-90 (sacrifices à Zeus Sôtèr de 334/3) et 118-119 (sacrifices à Zeus Sôtèr de 333/2) : respectivement 1050 et $2610 \frac{1}{2}$ drachmes, soit 150 et 377 bêtes. Je reprends l'estimation de 7 dr. par peau proposée par JAMESON (1988), p. 107-112, suivi par PARKER (2008), p. 37. Ces sacrifices à Zeus Sôtèr ne doivent pas être confondus avec les Dipolies-Bouphonies, contrairement à ce que pensait encore SCHMITT-PANTEL (1992), p. 140 qui suivait - à tort - Kirchner (IG).

80. Démosthène, Contre Timocrate, 121.

81. Lycurgue, Contre Léocrate: cf. supra, p. 104 ; Dinarque, Contre Démosthène, 36 ; Id., Contre Philoclès, 15. 


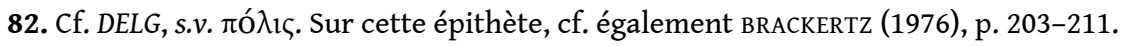

83. Cf. MIKALSON (1991), p. 210-213.

84. Cf. Tома (2008), p. 97-98.

85. Platon, Lois, XI, 921b-c. C'est peut-être sur la foi de ce passage qu'un scholiaste (Ulpien ?) à Démosthène, 21, 654, a intégré poliouchos à une liste d'épiclèses athéniennes du dieu (boulaios, phratrios, philios, herkeios) qui sont pour leur part bien attestées dans cette cité.

86. Cf. LECOMTE (1993), notamment p. 229-230 à propos de ce passage («Ce n'est pas d'Athènes qu'il est question ici, mais de la cité que Platon imagine en Crète »).

87. Sa figure n'a ainsi jamais été exploitée par le pouvoir impérial, notamment, contrairement à Samos, par exemple, où Auguste et Tibère lui ont été identifiés (IG XII 6, 499). À Athènes, c'est à Zeus Boulaios qu'Auguste a été associé (I.Eleusis 297).

88. Respectivement $I G I^{2}$, 5024 (kerkis centrale, juste à côté - jardin - de celui du prêtre de Dionysos Eleuthereus), 5025 (kerkis centrale, à deux sièges côté cour de celui du prêtre de Dionysos Eleuthereus) et 5063 (premier siège de la troisième kerkis côté cour en partant du centre) : cf. MAASS (1972), p. 104 et plan G III d, p. 105 et plan G I a, p. 129-130 et plan D II e.

89. Le rapport de force est différent à Cos, ce qui explique que Zeus Polieus et Athéna Polias (dans cet ordre) y forment un « vrai » couple : cf. PAUL (2010) et Id. (2013), p. 270-273 et 313-316.

\section{RÉSUMÉS}

L'examen de l'ensemble des données relatives au culte de Zeus Polieus à Athènes, tant dans l'asty que dans les dèmes (fin $\mathrm{du} \mathrm{VI}^{\mathrm{e}} \mathrm{s}$. - début du $\mathrm{III}^{\mathrm{e}} \mathrm{s}$. ap. J.-C.), permet de mettre en évidence trois dimensions de ce dieu : son ancrage fondamentalement acropolitain; sa position élevée, dont il tire de possibles compétences en matière agricole; son rôle politique. Toutefois, ce dernier aspect ne doit pas être surévalué : à Athènes, le Polieus n'est ni un dieu tutélaire ni un protecteur actif de la cité. Il semble plutôt garantir l'unité de la communauté politique et surtout la position tutélaire d'Athéna (Polias); c'est du moins ce que laisse entendre une glose d'Hésychios qui propose un récit étiologique des Bouphonies sensiblement différent de celui, bien connu, transmis par le De Abstinentia de Porphyre.

The study of the evidence regarding the cult of Zeus Polieus in Athens, in the asty as well as in the demes (end of $6^{\text {th }}$ c. - beginning of $3^{\text {rd }}$ c. CE), allows us to emphasize three main dimensions of the god. These are: his fundamental rooting on the Acropolis, the highness of his location - which explains his probable agricultural skills - and his political role. The Polieus' political capacity shall not be overestimated, though: in Athens, he is neither a tutelary god nor an active protector of the polis. He rather seems to guarantee the unity of the political community and, above all, the tutelary position of Athena (Polias). At least, this is what an aetiological tale for the Bouphonia, found in a gloss from Hesychios, seems to show. Indeed, this version of the myth differs noticeably from the more famous one from Pophyry's De Abstinentia. 


\section{AUTEUR}

\section{SYLVAIN LEBRETON}

Département des Sciences de l'Antiquité

Université de Liège / LAHM (UMR 6566 CReAAH, Rennes)

Sylvain.Lebreton@ulg.ac.be 